\title{
Adaptation of the International Affective Picture System (IAPS) for European Portuguese
}

\author{
Ana Paula Soares - Ana P. Pinheiro - Ana Costa • \\ Carla Sofia Frade • Montserrat Comesaña • Rita Pureza
}

Published online: 8 November 2014

(C) Psychonomic Society, Inc. 2014

\begin{abstract}
This study presents the results of the adaptation of the International Affective Picture System (IAPS) for European Portuguese (EP). Following the original procedure of Lang et al., 2000 native speakers of EP rated the 1,182 pictures of the last version of the IAPS set on the three affective dimensions of valence, arousal, and dominance, using the Self-Assessment Manikin (SAM). Results showed that the normative values of the IAPS for EP are properly distributed in the affective space of valence and arousal, showing the typical boomerang-shaped distribution observed in previous studies. Results also point to important differences in the way Portuguese females and males react to affective pictures that should be taken into consideration when planning and conducting research with Portuguese samples. Furthermore, the results from the cross-cultural comparisons between the EP ratings and the ratings from the American, Spanish, Brazilian, Belgian, Chilean, Indian, and BosnianHerzegovinian standardizations, showed that in spite of the fact that IAPS stimuli elicited affective responses that are similar across countries and cultures (at least in Western cultures), there are differences in the way Portuguese individuals react to IAPS pictures that strongly recommend the use of the normative values presented in this work. They can be downloaded as a supplemental archive at http://brm. psychonomic-journals.org/content/supplemental or at http:// p-pal.di.uminho.pt/about/databases.
\end{abstract}

A. P. Soares $(\bowtie) \cdot$ A. Costa $\cdot$ C. S. Frade $\cdot$ M. Comesaña $\cdot$ R. Pureza Human Cognition Laboratory, CIPsi, School of Psychology, University of Minho, Campus de Gualtar, 4710-057 Braga, Portugal e-mail: asoares@psi.uminho.pt

A. P. Pinheiro

Neuropsychophysiology Laboratory, CIPsi, School of Psychology, University of Minho, Braga, Portugal
Keywords Affective picture stimuli $\cdot$ IAPS $\cdot$ Emotion Valence $\cdot$ Arousal $\cdot$ Dominance $\cdot$ European Portuguese

\section{Introduction}

The study of emotions has attracted the interest of an increasing number of researchers in the last decades, as demonstrated by the exponential number of publications aiming to investigate the neurophysiological correlates of emotional processing (e.g., Bradley, Hamby, L w, \& Lang, 2007; Cuthbert, Schupp, Bradley, Birbaumer, \& Lang, 2000; Lang, Bradley, \& Cuthbert, 1998a; Liu, Pinheiro, Deng, Nestor, McCarley, \& Niznikiewicz, 2012; Paulmann \& Kotz, 2008; Pinheiro et al., 2013a), as well as to explore the influence of emotion on cognitive processes such as attention (e.g., Fox, Griggs, \& Mouchlianitis, 2007; Schimmack, 2005), memory (e.g., Bradley, Greenwald, Petry, \& Lang, 1992; Kensinger, \& Schacter, 2006; Mickley \& Kensinger, 2008), or language (e.g., Comesaña, Soares, Perea, Piñeiro, Fraga, \& Pinheiro, 2013; Scott, O'Donnell, Leuthold, \& Sereno 2009). Overall, these studies confirm the privileged status of emotional over neutral stimuli, which highlights the pervasive influence of emotion on human life.

The development of reliable studies on emotional processing requires the existence of standardized stimuli. Researchers have used different types of emotionally evocative stimuli, such as words (e.g., the Affective Norms of English Words [ANEW] - Bradley \& Lang, 1999a; the Berlin Affective Word List [DENN-BAWL] - Briesemeister, Kuchinke, \& Jacobs, 2011; the French Emotional Evaluation List [FEEL] - Gilet, Grühn, Studer, and Labouvie-Vief 2012), sounds (e.g., the International Affective Digitized Sounds [IADS] - Bradley \& Lang, 1999b, 2007a; the Montreal Affective Voices Belin, Fillion-Bilodeau, \& Gosselin, 2008; the Chinese Vocal Emotional Stimuli - Liu \& Pell, 2012), or pictures 
(e.g., the International Affective Picture System [IAPS] Lang, Bradley, \& Cuthbert, 1995-2008; the Karolinska Directed Emotional Faces [KDEF] - Lundqvist, Flykt, \& Öhman, 1998; the Japanese and Caucasian Facial Expressions of Emotion [JACFEE] - Ekman \& Matsumoto, 1993-2004; the Montreal Set of Facial Displays of Emotion Beaupre, Cheung, \& Hess, 2000; the Geneva Affective Picture Database [GAPED] - Dan-Glauser \& Scherer, 2011; and the Nencki Affective Picture System [NAPS] - Marchewka, Żurawski, Jednoróg, \& Grabowska, 2014).

Despite the diversity of emotional evocative stimuli in the literature, pictures have been selected much more often than sounds or verbal stimuli (e.g., Cuthbert et al., 2000). Studies testing different types of emotional evocative stimuli suggest a differential access to affective information as well as differences in the neural networks activated as a function of the type of stimuli used (e.g., De Houwer \& Hermans, 1994; Hinojosa, Carretie, Valcarcel, Mendez-Bertolo, \& Pozo, 2009; Kensinger \& Schacter, 2006; Potter, Staub, \& O'Connor 2004). For example, pictures were processed faster (e.g., De Houwer \& Hermans, 1994) and were better remembered than words (e.g., Potter et al., 2004), which might indicate that pictures are more biologically relevant than words and less semantically mediated (Hinojosa et al., 2009). Furthermore, since pictures represent static stimuli (contrary to sounds) and have minimal linguistic confounds (contrary to verbal stimuli), these features make them particularly suited for cognitive and neurophysiological research on affective processing, and simultaneously allow an easier comparison of results across different languages/cultures.

Within the available sets of standardized pictures, the IAPS (Lang et al., 1995-2008) is one of the most widely used set of stimuli in research aiming, for example, to investigate differences in the behavioral and neural correlates underlying the processing of neutral versus emotional pictures (e.g., Bradley et al., 2007; Calvo \& Avero, 2009; Cuthbert et al., 2000; Lang et al., 1998a, b), the effects of mood elicited by pictures on cognitive processing (e.g., Pinheiro, Liu, Nestor, McCarley, Goncalves, \& Niznikiewicz, 2013b; Van Berkum, De Goede, Alphen, Mulder, \& Kerstholt, 2013), the effects of arousal and valence on perception (e.g., Carretié, Mercado, Tapia, \& Hinojosa, 2001; Cuthbert et al., 2000; Liu et al., 2012), and on immediate and long-term memory tasks (e.g., Bradley et al., 1992; Kensinger, \& Schacter, 2006; Mickley \& Kensinger, 2008), as well as on the study of developmental differences in emotion processing (e.g., Grühn \& Scheibe, 2008; McManis, Bradley, Berg, Cuthbert, \& Lang, 2001), or on the characteristics of emotion processing in clinical groups (e.g., Horan, Wynn, Kring, Simons, \& Green, 2010; Pinheiro et al., 2013b; Tok, Koyuncu, Dural, \& Catikkas, 2010).

Several factors may justify the preference for this stimulus set in affective research: (a) it is one of the first pictures set developed with the aim of promoting research in affective processing by the Center for Emotion and Attention (CSEA,
University of Florida); (b) since its first version, this set has incorporated a wide range of color pictures from different content categories (e.g., faces, animals, landscapes, people, objects, erotic scenes or mutilation) and has been updated with a growing number of pictures in its successive versions (from 1995 to 2008; Lang et al., 1995-2008); (c) it is a reliable measure that is based on a clear theoretical account of emotions (dimensional) (e.g., Bradley \& Lang, 1994; Bradley, Codispoti, Sabatinelli, \& Lang, 2001a; Osgood, Suci, \& Tannenbaum, 1957; Russell, 1980), as we will discuss later in the manuscript; (d) the affective ratings of its pictures strongly correlate with different peripheral physiological responses such as skin conductance (e.g., Bradley \& Lang, 2000; Codispoti, Ferrari, \& Bradley, 2006), startle response (e.g., Bradley, Cuthbert, \& Lang, 1999; Bradley, Miccoli, Escrig, \& Lang, 2008), and heart rate (e.g., Bradley \& Lang, 2000); (e) since the original North American version (Lang et al., 1995), IAPS norms have been adapted to a growing number of countries/cultures (Spain: Moltó et al., 1999; Vila et al., 2001; Belgium: Verschuere, Crombez, \& Koster, 2001; Mexico: Castillo-Parra, De Jesús, Ostrosky-Solís, \& Ostrosky-Solís, 2002; China: Shao-hua, Ning, \& Wen-tao, 2005; Yi, Liu, Luo, \& Yao, 2006; Yuxia \& Yuejia, 2004; Brazil: Lasaitis, Ribeiro, \& Bueno, 2008; Ribeiro, Pompéia, \& Bueno, 2005; Bosnia-Herzegovina: Drače, Efendić, Kusturica, \& Landžo, 2013; Hungary: Déak, Csenki, \& Révész, 2010; Germany: Barke, Stahl, \& Kröner-Herwig, 2012; Grühn \& Scheibe, 2008; Chile: Dufey, Fernández, \& Mayol, 2011; Silva, 2011; India: Lohani, Gupta, \& Srinivasan, 2013); (f) it is part of a wider system for emotion assessment that additionally includes a set of emotional words (ANEW Bradley \& Lang, 1999a) and emotional sounds (IADS Bradley \& Lang, 1999b, 2007a).

However, in spite of the relevance of the IAPS for research on emotion and attention, no normative ratings were yet available for European Portuguese (EP). This represented an important obstacle for research with EP participants. Responding to that need, the aim of this study was to obtain Portuguese norms for the most recent version of the IAPS (Lang et al., 2008) set. These norms complete the adaptation of the three sets of affective stimuli developed by the CSEA (ANEW - Bradley \& Lang, 1999a; and IADS -2007a) for EP (Soares, Comesaña, Pinheiro, Simões, \& Frade, 2012; Soares, Pinheiro, Costa, Frade, Comesaña, \& Pureza, 2013, respectively), which represents an important contribution to the cognitive and psychophysiological research in the domain of emotional processing in Portugal. In particular, it will allow researchers to control and/or manipulate the affective properties of stimuli to be used both in unimodal and in multimodal paradigms, and also to develop cross-linguistic studies matching stimuli in the same affective dimensions in the languages and cultures for which normative values are also available. 
The last version of the IAPS (Lang et al., 2008) includes 1,182 pictures depicting a wide range of content categories such as animals, natural landscapes, social scenes, erotic scenes, or mutilation. Based on a dimensional account of emotions, which proposes that emotions should be characterized along a small number of underlying and independent dimensions (e.g., Bradley \& Lang, 1994; Osgood et al., 1957; Russell, 1980), the IAPS set provides, for each picture, norms for the affective dimensions of valence (indexing the degree of pleasantness that a stimulus can generate, ranging from something that is unpleasant and makes the participant feel sad or unhappy to something that is pleasant and makes him/her feel happy), arousal (indexing the degree of excitement or activation a subject can feel towards a stimulus, ranging from feeling calm and relaxed to feeling exCitation or being alert) and dominance (indexing the degree of control a subject feels over a stimulus, ranging from the feeling that he/she has no power to handle the situation to feeling dominant or in control of it). The ratings for each of these affective dimensions were collected using the SelfAssessment Manikin (SAM - Bradley \& Lang, 1994). The SAM is a non-verbal pictorial technique for assessment of emotions in which each affective dimension of valence, arousal, and dominance is represented by 5 figures inserted in a 9point scale (as illustrated in Fig. 1). Participants were asked to choose the value of the 9-point scale that best represented their affective reaction to the emotionally evocative stimuli presented in each of the three affective dimensions. The SAM scales are a reliable measure of emotion (correlating strongly, as mentioned before, with different peripheral physiological measures (e.g., Bradley \& Lang, 2000; Bradley et al., 1999,
2008; Codispoti et al., 2006) and has been assumed as the standardized procedure to collect ratings and provide norms of valence, arousal, and dominance of the IAPS set in all the international standardizations developed so far.

Bradley and collaborators (Bradley \& Lang, 1994; Bradley et al., 2001a) argue that the assessment of each of these dimensions organizes the response of the individuals to affective stimuli, which can be conceptualized considering two fundamentally motivational systems of avoidance (the defensive system) and approach (the appetitive system). The defensive system is primarily activated in contexts representing a threat to an organism's survival, eliciting behaviors such as withdrawal or attack, while the appetitive system is primarily activated in contexts that promote the well-being and survival of the organism. These two systems account for two basic dimensions of emotion: valence and arousal. The first indicates which system is active, while the second reflects the intensity of the activation. Therefore, the emotional reaction elicited by affective stimuli may be described by its location on a two-dimensional affective space (ordinate = valence; abscissa $=$ arousal). Typically, the distribution of the IAPS ratings in the affective space is boomerang-shaped, given that neutral stimuli tend to receive low arousal scores, while positively and negatively valenced stimuli tend to be rated as more arousing (e.g., Bradley et al., 2001a; Lang et al., 1998a, b). A third dimension was additionally proposed by Bradley and Lang (1994) - dominance, indexing, as abovementioned, the level of control that a stimulus evokes. It is also worth noting that besides this dimensional characterization, the IAPS pictures were also described in terms of different discrete categories, based on a categorical account
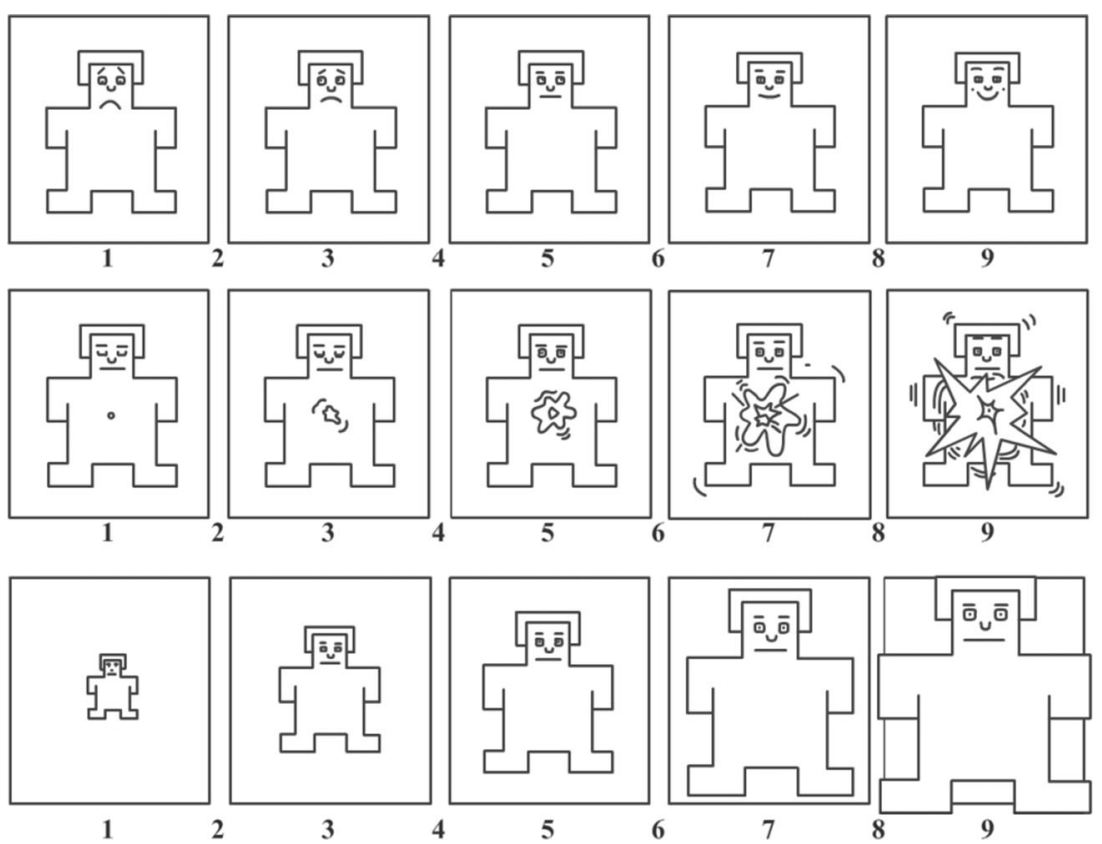

Fig. 1 Self-Assessment Manikin (SAM) used for the assessment of the valence (top), arousal (middle), and dominance (bottom) affective dimensions. The five figures in each affective dimension and the spaces between them define the 9-point scale that was used 
of emotion (see Barke et al., 2012; Davis et al., 1995; Libkuman, Otani, Kern, Viger, \& Novak, 2007; Mikels, Fredrickson, Larkin, Lindberg, Maglio, \& Reuter-Lorenz, 2005 for details). The combination of these two perspectives in the characterization of each IAPS picture makes this set of standardized picture stimuli a powerful resource to support current research on affective processing based both in a dimensional and/or categorical conceptual framework.

Since it represents a resource of excellence for studies on attention and affective processing, it is not surprising that there were several attempts to validate the IAPS norms to different languages and cultures. For example, Moltó et al. (1999), in one of the first empirical works with that aim, provided norms for 480 pictures from the 1999 IAPS version for Spanish. This first Spanish work was then complemented by Vila et al. (2001), whose work provided IAPS norms for an additional 352 pictures. In Belgium, Verschuere et al. (2001) conducted the Flemish validation of the IAPS set, which provides affective norms for 60 pictures that were selected by using a stratification procedure from the total set of the 1995 IAPS version. Moreover, in Brazil, Ribeiro et al. (2005) provided norms for 707 pictures of the 1999 IAPS version. Thereafter, Lasaitis et al. (2008) continued this initial work for Brazilians, providing norms for more 240 IAPS pictures. More recently, Déak et al. (2010) provided affective ratings for 239 pictures of the 2005 IAPS version for Hungarian, while Dufey et al. (2011) and Silva (2011) provided norms for respectively 188 and 118 IAPS pictures for Chilean participants from the 2005 version. In Germany, Grühn and Scheibe (2008) reported data for 504 IAPS pictures of the 1998 IAPS version rated by younger and older adults, and Barke et al. (2012) provided affective ratings for 298 IAPS pictures, combining both dimensional and categorical measurements. Lohani et al. (2013) in India provided normative ratings for 100 pictures of the 2005 IAPS version. Finally, in Bosnia and Herzegovina, Drače et al. (2013) provided recently norms for 60 pictures from the 1995 IAPS version.

In the present work, we provide norms of valence, arousal, and dominance for all pictures $(N=1,182)$ of the last version of the IAPS (Lang et al., 2008) set. Following our previous studies (Soares et al., 2012, 2013), we also explored sex differences in IAPS ratings by EP participants. The investigation of sex differences is encouraged by recent studies (e.g., Bradley, Codispoti, Sabatinelli, \& Lang, 2001b; Hamann, Herman, Nolan, \& Wallen, 2004; Karama et al., 2002; Kemp, Silberstein, Armstrong, \& Nathan, 2004; Lithari et al., 2010; Pessoa, 2009; Wrase et al., 2003) and by our previous work demonstrating differences in how male and female participants rate affective words (Soares et al., 2012) and sounds (Soares et al., 2013). For example, recent studies show that women tend to respond with greater defensive reactivity to negative visual stimuli (i.e., with higher psychological and physiological activation), while men tend to respond with greater appetitive reactivity to positive stimuli, particularly to erotic stimuli (Bradley et al., 2001b). Sex differences in the response to erotic stimuli were also confirmed by functional magnetic resonance imaging ( $f M R I)$ studies, indicating stronger amygdala and hypothalamus activation to erotic stimuli in males relative to females (e.g., Hamann et al., 2004; Karama et al., 2002). Male participants also showed lower inhibitory control in tasks involving the previous presentation of erotic stimuli (e.g., Pessoa, 2009; Yu et al., 2012). Furthermore, increased frontal lobe activation to positive visual stimuli was found in men relative to women, while increased activation in the cingulate gyrus (anterior and medial) to negative visual stimuli was found in women relative to men (Wrase et al., 2003). Other studies pointed to electrophysiological differences in the processing of affective pictures, such as evinced by the increased amplitude of N100 and N200 components to negative visual stimuli in females relative to males (Lithari et al., 2010), and reduced latency of frontal steady-state visually evoked potentials related to the processing of negative visual stimuli in females but not in males (Kemp et al., 2004). Taken together, these findings suggest important behavioral and brain differences in the way men and women process emotional stimuli, which strongly recommends the analysis of sex effects on IAPS ratings. In line with the abovementioned studies, we expected to observe sex differences in IAPS ratings by EP participants, with females being more emotionally reactive than males (i.e., reacting with higher levels of arousal and using more extreme valence values when rating pleasant and unpleasant IAPS pictures), even though we expected men to show higher scores of dominance and to rate erotic pictures as more positive and more arousing than women.

Moreover, in this work we explored crosscultural differences to determine whether Portuguese subjects rated IAPS pictures similarly to subjects from the countries and culturesfor which we had access to se normative values (i.e., American, Belgian, Brazilian, Bosnia-Herzegovinian, Chilean, Hungarian, Indian, and Spanish standardizations). In spite of the fact that the IAPS pictures elicited emotional responses that are similar in different countries and cultures (e.g., Déak et al., 2010; Drače et al. 2013; Dufey et al. 2011; Moltó et al., 1999; Lasaitis et al., 2008; Ribeiro et al., 2005; Silva, 2011; Verschuere et al., 2001; Vila et al. 200), some differences between the American and other standardizations have been observed. For example, Lohani et al. (2013) found that Indian subjects rated IAPS pictures with higher levels of arousal and dominance than the USA counterparts; Barke et al. (2012) found that German subjects rated the IAPS pictures as more positive and less arousing than Americans; Moltó et al. (1999) and Vila et al. (2001) found that Spanish participants perceived IAPS pictures as more arousing and less dominant than Americans; and Ribeiro et al. (2005) and Lasaitis et al. (2008) found that Brazilian subjects rated IAPS 
pictures with higher levels of arousal than Americans, a result also observed by Dufey et al. (2011) in a study with Chilean subjects.

The crosscultural differences observed when comparing the IAPS normative values in different countries and cultures provide support for the influence of culture on the way individuals perceive, feel, and express emotions (see Mesquita \& Walker, 2003) and justify the need to explore whether Portuguese participants differ from American subjects in how they rate IAPS pictures, following the same strategy adopted in our previous studies (Soares et al., 2012, 2013). This analysis will contribute to the crosscultural validity of the IAPS set. Considering our previous studies (Soares et al., 2012, 2013), we expected Portuguese participants to be less emotionally reactive to IAPS pictures when compared with American and Spanish participants (particularly in what concerns the arousal affective dimension), even though the absence of previous comparative studies do not allow us to formulate more specific hypotheses when comparing Portuguese ratings with the ratings obtained from Belgian, Brazilian, Bosnia-Herzegovinian, Chilean, Hungarian, and Indian participants.

\section{Method}

\section{Participants}

Two thousand undergraduate students (1,419 females and 581 males; $M_{\text {age }}=21.57 ; S D=5.67$; female sample: $M_{\text {age }}=21.19$; $S D=5.30$; male sample: $M_{\text {age }}=22.51 ; S D=6.38$ ) from different undergraduate programs (Humanities, Economics, Sciences, and Technologies) in Portuguese universities participated in the study. ${ }^{1}$ This sample excluded participants whose native language and/or nationality was not Portuguese $(N=$ $124)$, as well as those who did not respond to more than $20 \%$ of the items $(N=30)$, or whose responses showed nondiscriminative ratings and suggested random responses or inattention (e.g., choosing the same number for all pictures $N=22$ ). The majority of the participants had normal (72\%) or corrected-to-normal visual acuity $(28 \%)$.

\section{Materials and procedure}

In order to compute the normative values of valence, arousal, and dominance of the IAPS set for EP, we used the last version of this set that includes 1,182 pictures (Lang et al., 2008).

\footnotetext{
${ }^{1}$ Specifically from the University of Minho, University of Porto, University of Beira Interior, Universidade de Trás-os-Montes e Alto Douro, and University of Aveiro, University of Coimbra, University Católica Portuguesa, and University of Lisbon.
}

These pictures were distributed in 20 sets of approximately 60 pictures according to the original normative study of Lang et al. (2008). Participants rated only one set of pictures that was randomly assigned. Each set was rated by 100 subjects, with a male:female ratio that approached 1:3 in each set.

The ratings were collected in a laboratory setting in groups that did not exceed ten participants per session. After explaining the aims of the study and obtaining written consent from participants, the instructions for the affective rating task were presented. Data collection followed the procedure described by Lang et al. (2008). Specifically, participants were told that during the experiment approximately 60 pictures would be presented and were asked to rate each of them in terms of "how it made them feel while observing it" by using a paperand-pencil version of the SAM scales (Bradley \& Lang, 1994). Figure 1 illustrates the SAM scales used in the present study.

As mentioned before, the SAM scales are a 9-point nonverbal pictorial rating system designed to obtain selfassessments of the affective reactions of individuals in the dimensions of valence, dominance, and arousal (Bradley \& Lang, 1994). The valence scale is represented at the top of Fig. 1 by a frowning, unhappy face (value " 1 " in the valence scale) changing to a smiling, happy face (" 9 " in the valence scale), describing the negative (unpleasant) or positive feeling (pleasant) that the situation, object, or event depicted by the picture generates in the individual. Arousal is another scale that is represented at the middle of Fig. 1 by a sleepy closedeyes face (" 1 " in the arousal scale) changing to an excited open-eyes face ("9" in the arousal scale). It describes the perceived vigilance as a physiological and psychological response to the picture, ranging from feeling calm and relaxed to feeling excitation or being in alert. Finally, the dominance scale is represented at the bottom of Fig. 1 by a very small figure ( " 1 " in the dominance scale) changing to a very large figure ("9" in the dominance scale) that describes how much the participant feels being in or out of control of the situation, object, or event depicted by the picture.

For each IAPS picture participants were asked to choose a number from the 9-point scale that best represented the way they felt while observing it in each of the three affective dimensions. They were also instructed that neutral responses to the stimuli that, for example, in the valence scale generated feelings of neither happiness nor sadness, should be signaled by the choice of the number 5 . The same value should be chosen if pictures did not make the participants feel either "relaxed" or "in alert" in the arousal scale, and neither "controlled" nor "in control" in the dominance scale. Participants were asked to pay the maximum attention to each picture before rating it in each of the three affective dimensions in the SAM scales. They were also asked to respond as quickly and honestly as possible.

Prior to the affective ratings of the experimental stimuli, pictures 4200 [woman at beach], 7010 [basket], and 3100 [a 
burn victim] were used as practice items, as in the IAPS normative study (see Lang et al., 2008). After completing the practice items, the experimental session began with the distribution of a booklet to each participant. The booklet contained questions asking participants to fill out sociodemographic and linguistic information (e.g., sex, age, nationality, lateralization, visual acuity, educational level, native language). It also contained the numerical codes of the pictures included in the set to be rated, and the paper-andpencil SAM scales (see Fig. 2c).

Participants were then seated separately in a quiet room in front of a computer. Pictures were presented in their original size $(1024 \times 768)$ at the center of a 17 -inch computer screen with a $1280 \times 1024$ resolution and at a distance of approximately $60 \mathrm{~cm}$. The software SuperLab Pro 4.5 (Cedrus Corporation, San Pedro, CA, USA) was used for stimuli presentation. It also allowed the randomization of pictures in each set per participant. Each set was composed of approximately 60 trials. The structure of a trial for any of the 20 sets is illustrated in Fig. 2.

Before viewing a given IAPS picture (the number 2550 was used in Fig. 2 as a hypothetical example), the instruction: "Por favor avalie a próxima imagem na linha número $n$ [Please rate the next picture in line number $n$ ]" appeared in the center of the screen (Arial font, 14) for $5 \mathrm{~s}$ (see Fig. 2). During that period, participants searched in the booklet for the numerical code that corresponded to the row where that picture should be rated in the three affective dimensions of valence, arousal, and dominance (e.g., picture 2550, as illustrated in Fig. 2). After the 5-s period elapsed, the IAPS picture was presented for $6 \mathrm{~s}$. During that time, participants were instructed to pay maximum attention to the picture. Then, the instruction: "Por favor avalie agora a imagem nas três dimensões afectivas [Please rate the picture in the three affective dimensions now]" was presented at the center of the screen (Arial font 14) for $15 \mathrm{~s}$. During that time participants were to rate the picture observed in the three affective dimensions in the row of their booklet previously identified. When the response time has elapsed, the next trial began. Each trial lasted $26 \mathrm{~s}$ and the entire procedure took approximately 30 min per participant.

\section{Results and discussion}

The EP normative values of valence, arousal and dominance of the IAPS set (Lang et al., 2008) can be downloaded as a supplemental archive at http://brm.psychonomic-journals.org/ content/supplemental or at http://p-pal.di.uminho.pt/about/ databases. The supplemental archive shows the mean values $(M)$ and standard deviations $(S D)$ for valence $(\mathrm{Val})$, arousal (Aro), and dominance (Dom) of each of the 1,182 pictures included in the adaptation of the IAPS to $\mathrm{EP}^{2}$ considering the total sample (All) as well as the subsamples of females $(\mathrm{Fem})$ and males (Mal) separately. Pictures were organized considering their original number (Number) in the IAPS set (Lang et al., 2008). After the number, the original English description (E-description) and the EP description (EPdescription) are presented. The number of the set in which each picture was included, both in the American and in the Portuguese normative studies, is also provided (Set).

The results of the normative study of the IAPS pictures (Lang et al., 2008) to EP are presented in two different sections. First, we present the distribution of the EP ratings in the bi-dimensional affective space of valence and arousal and explore sex differences in the way Portuguese females and males rated the IAPS pictures. Secondly, we analyze crosscultural differences in the way Portuguese participants and participants from other countries and cultures rated IAPS pictures. As previously mentioned, in this analysis we considered the normative values from the last version of the American (USA), the Spanish (SP), the Brazilian (BR), the Belgian (BG), the Chilean $\left(\mathrm{CH}_{1}\right.$ and $\left.\mathrm{CH}_{2}\right)$, the Indian (IND), and the Bosnia-Herzegovinian (BH) IAPS standardizations. It is worth noting that even though the comparison between the EP and the USA standardizations considers the normative values obtained for all the pictures of the 2008 IAPS version $(N=1,182)$, the comparison with the other standardizations included a lower number of pictures, since those works were based on older versions of the IAPS set (1995, 1999 and 2005) that had a fewer number of pictures.

Specifically, the comparison with the SP standardization considers 820 common pictures from both Moltó et al. (1999) and Vila et al. (2001) studies. The comparison with the BR standardization considers 916 common pictures from both the Lasaitis et al. (2008) and the Ribeiro et al. (2005) studies. The comparison with the $\mathrm{BG}$ and the $\mathrm{BH}$ standardizations considers 60 common pictures from the Verschuere et al. (2001) and the Drače et al. (2013) studies, respectively, and the comparison with the IND version considers 100 common pictures from the Lohani et al. (2013) study. Finally, the comparison with the Chilean standardizations considers 188 common pictures from the Dufey et al. (2011) study $\left(\mathrm{CH}_{1}\right)$ and 118 common pictures from the Silva (2011) study $\left(\mathrm{CH}_{2}\right)$ since then $\mathrm{CH}_{1}$ study only provides data for the total sample, and the $\mathrm{CH}_{2}$ study only provides data for males and females separately. Furthermore it is worth noting that the crosscultural analyses between EP and the $\mathrm{CH}_{1}$ and $\mathrm{CH}_{2}$

\footnotetext{
${ }^{2}$ It is worth noting that the supplemental archive presents affective ratings for 1,194 pictures, similar to the USA set (Lang et al., 2008), since pictures 1230, 1590, 1610, 1640, 1670, 2210, 3000, 3010, 4520, 6200, 6571 , and 9090 were associated with two different sets. Thus, the normative values of valence, arousal, and dominance of those pictures were provided twice, each time for a different set, as in Lang et al.'s (2008) normative study.
} 


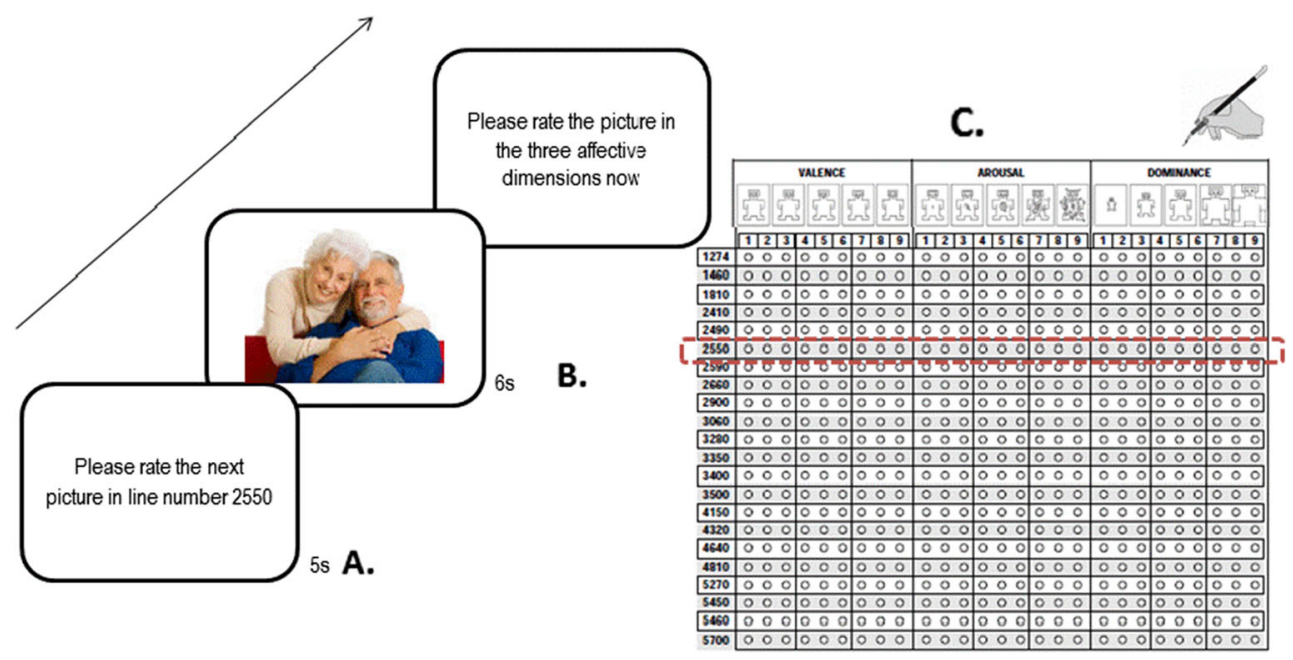

Fig. 2 Example of an experimental trial of the affective rating task. The picture presented is not part of the International Affective Picture System (IAPS) set and was selected only to serve as a stimulus example (obtained from FreeDigitalPhotos.net available at: http://www.freedigitalphotos. net/images/People_g40.html). (A) The participant reads the instruction: "Please rate the next picture in line number 2550" and identifies the row that corresponds to that number (2550) in the response sheet (5s); (B) The

standardizations were only conducted for the valence and arousal affective dimensions (since dominance ratings were not provided in both works) and the EP comparisons with the $\mathrm{BG}$, IND, and BH standardizations were also only conducted for the total sample of participants (since those works do not provide norms for males and females separately, as the $\mathrm{CH}_{1}$ standardization). Nevertheless and in spite of the lower number of pictures, dimensions, and/or samples that each of those standardizations provided, we considered that the inclusion of those works in the validation of the EP IAPS set may enrich the analysis of how people from different countries and cultures react to the same affective stimuli, thus contributing for the crosscultural validation of the IAPS set.

\section{EP ratings of IAPS stimuli}

Figure 3 illustrates the distribution of EP ratings (mean values) for 1,182 IAPS pictures in the bi-dimensional affective space of valence and arousal, considering data from the total Portuguese sample, i.e., males and females together. ${ }^{3}$

The distribution presented in Fig. 3 showed that the EP ratings of the IAPS pictures fits the typical boomerang-shape reported by Lang et al. in the successive USA versions of the IAPS (1995-2008), as well as observed in the other international standardizations (e.g., Drače et al., 2013; Dufey et al., 2011; Lasaitis et al., 2008; Moltó et al., 1999; Ribeiro et al.

\footnotetext{
$\overline{3}$ Since 12 pictures are associated with two different sets in the original and in our dataset (pictures 1230, 1590, 1610, 1640, 1670, 2210, 3000, $3010,4520,6200,6571$, and 9090), in this analysiss we included the normative values of these pictures only once to avoid the existence of different values for the same IAPS stimuli.
}

participant observes the picture corresponding to the numeric code presented at the center of the computer screen during $6 \mathrm{~s}$; and $(C)$ The participant reads the instruction: "Please rate the picture in the three affective dimensions now" and rates the picture in the three affective dimensions of valence, arousal, and dominance by using the SelfAssessment Manikin (SAM) scale in the response sheet (15 s). This procedure was repeated for each picture included in the set

2005; Verschuere et al., 2001; Vila et al., 2001; Silva, 2011). As illustrated in Fig. 3, the mean scores of the IAPS pictures are well distributed along two axes stretching from the valence score of 5 points (the midpoint of neutrality in the 9-point scale used). It is worth noting that despite the fact more recent studies use a more extended interval that includes the categorization of neutral stimuli (ranging usually from 4 to 6 points), the value 5 was used as cutoff in the classification of pleasant (i.e., pictures with valence scores above 5 points) and unpleasant (i.e., pictures with valence scores below 5 points) pictures. This option was motivated by the fact that it is the criterion adopted by Bradley and Lang to define the two motivational systems of avoidance (the defensive system) and approach (the appetitive system), and the same criterion was adopted by all the existing normative studies of the IAPS set (e.g., Drače et al., 2013; Dufey et al., 2011; Lang et al., 1995-2008; Lasaitis et al., 2008; Moltó et al., 1999; Ribeiro et al. 2005; Verschuere et al., 2001; Vila et al., 2001; Silva, 2011).

Thus, similar to the results observed in all the other IAPS standardizations (e.g., Drače et al., 2013; Dufey et al., 2011; Lasaitis et al., 2008; Moltó et al., 1999; Ribeiro et al. 2005; Verschuere et al., 2001; Vila et al., 2001; Silva, 2011), the present results showed that highly pleasant (i.e., pictures with valence scores near the maximum of the 9-point scale) and highly unpleasant pictures (i.e., pictures with valence scores near the minimum of the 9-point scale) tend to be rated as highly arousing, resulting in a quadratic relationship $(r=.70$, $\left.R^{2}=.49, p<.001\right)$ that is superior to its pairwise linear correlations $\left(r=.68, R^{2}=.46, p<.001\right)$. It is worth noting that this quadratic correlation was higher and explains more variance (49\%) than what was observed in all the other 


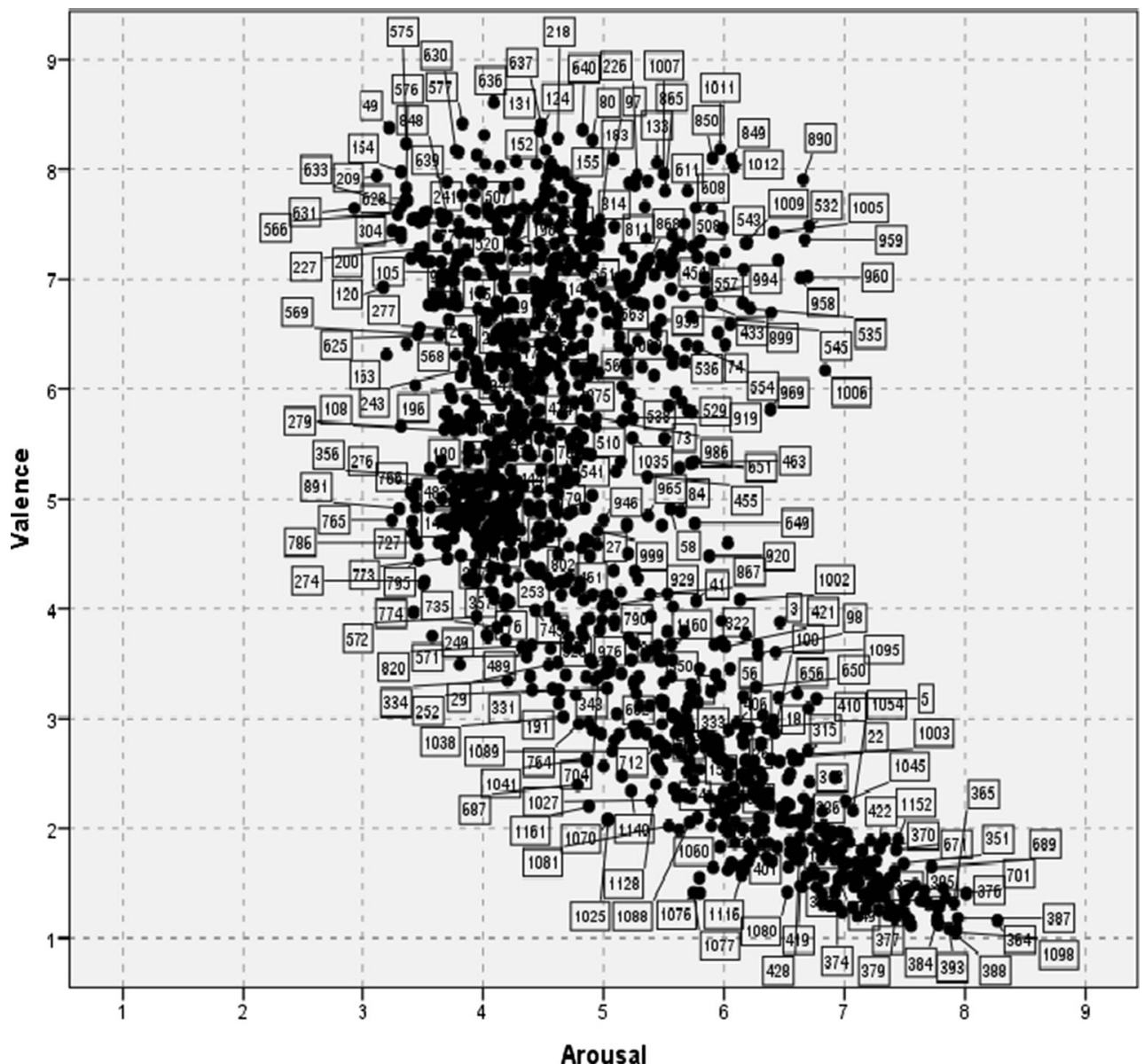

Fig. 3 Distribution of mean values for the valence and arousal affective dimensions in the total sample of the European Portuguese (EP) standardization of the International Affective Picture System (IAPS)

international IAPS standardizations (ranging from $11 \%$ in the USA standardization to $48 \%$ in the $\mathrm{CH}_{1}$ standardization, as presented later in the crosscultural analysis section), with the exception of the BR standardization (71\%).

Additionally, analysis of the EP results showed that even though the association between valence and arousal is statistically significant both for the appetitive and defensive motivational systems $(p<.001)$, the correlation is stronger for the defensive $(r=-.89)$ than for the appetitive $(r=.13)$ system. As shown in Fig. 3, most of the pleasant pictures (that are located in the upper half of the chart) are more concentrated around the midpoint of the arousal scale $(M=4.52, S D=.71$, range $=3.92)$ and present lower dispersion than unpleasant pictures (that are located in the lower half of the chart) $(M=$ $5.61, S D=1.19$, range $=5.02$ ). This finding suggests that Portuguese subjects primarily rate unpleasant IAPS pictures as more arousing than pleasant pictures. For example, pictures 3053 [BurnVictim], 3131 [Mutilation], 3001 [HeadlessBody], and 6550 [Attack] were rated as simultaneously the most negative $\left(M_{\text {valence }}=1.08,1.08,1.16,1.41\right.$, respectively $)$ and the most arousing pictures in the EP set $\left(M_{\text {arousal }}=7.93,7.87\right.$, $8.27,8.01$, respectively). However the same was not observed for pleasant pictures. For example, pictures 5825 [Sea], 5210 [Seaside], 5829 [Sunset], and 1441 [PolarBears] were rated with the highest valence scores $\left(M_{\text {valence }}=8.61,8.42,8.41\right.$, 8.38 , respectively), but not with equivalent arousal scores $\left(M_{\text {arousal }}=4.09,3.83,4.48,3.22\right.$, respectively $)$. This seems to be the case only for a small subset of stimuli associated with erotic content (e.g., pictures 4652 [EroticCouple], 4651 [EroticCouple]) or with radical sports (e.g., pictures 8179 [Bungee], 8178 [Cliffdiver]), which were both rated with high scores in valence $\left(M_{\text {valence }}=7.49,7.17,7.36,7.01\right.$, respectively) and arousal ( $M_{\text {arousal }}=6.71,6.45,6.67,6.64$, respectively). Nonetheless, it is important to note that the arousal ratings observed for those pictures are still lower than those observed for the negative IAPS pictures.

The stronger relationship between valence and arousal in the defensive than in the appetitive motivational system in the EP adaptation of the IAPS reflects the negativity bias observed in previous studies (e.g., Bradley \& Lang, 2007b; Bradley et al., 2001a; Dufey et al., 2011; Bradley \& Lang, 1999b, 2000; Moltó et al., 1999; Ribeiro et al., 2005; Soares et al., 2012, 2013; Verschuere et al., 2001; Vila et al., 2001), and shows that Portuguese subjects are more emotionally 
reactive to unpleasant than to pleasant IAPS pictures. This negativity bias is also observed if we assume, as for the valence dimension, 5 as the cutoff value in the classification of high-arousing (above 5) and low-arousing (below 5) pictures. The distribution of the EP ratings attending both to valence and arousal scores showed that, even though there is a relatively balanced number of pictures classified as negative and as positive in the EP of the IAPS set (597 vs. 585, respectively), there is a higher number of unpleasant pictures classified as high (390) than as low (207) arousing, whereas for the pleasant ones there is a higher number of pictures classified as low (459) than as high arousing (126), $\chi^{2}(1)=$ 239.32, $p<.001$.

This result reveals that, similar to the American (Lang et al., 2008) and other international standardizations (e.g., Drače et al., 2013; Dufey et al., 2011; Lasaitis et al., 2008; Moltó et al., 1999; Ribeiro et al. 2005; Verschuere et al., 2001; Vila et al., 2001; Silva, 2011), it is easier to find unpleasant than pleasant pictures with higher scores of arousal in the EP adaptation of the IAPS. This result may hinder research using pleasant pictures with EP participants when the manipulation of the arousal is intended. Thus, future extensions of this set should consider the possibility of increasing the number of pleasant high-arousing stimuli by including, for example, a higher number of erotic and radical sports pictures that, as noted before, tend to be rated with higher scores of arousal. Nevertheless, the dispersion of results observed both for valence $(\min =1.05, \max =8.61)$ and arousal $(\min =2.92, \max =$ 8.27) in the EP set provides Portuguese researchers with a diversified pool of stimuli that allow the control and/or the manipulation of the affective properties of pictures for different research purposes.

Table 1 presents means, standard deviations, and range values of the Portuguese IAPS ratings for each of the affective dimensions of valence, arousal, and dominance for all the pictures and for pleasant and unpleasant pictures (classified on the basis of the global sample ratings), considering the total sample (all) and the subsamples of females (fem) and males (mal) separately.

In order to explore sex differences in the way Portuguese females and males rated IAPS pictures, we conducted a multivariate analysis of variance (MANOVA) on the mean scores obtained for each picture, considering sex (females vs. males) and picture valence (unpleasant vs. pleasant) as betweensubject factors and the ratings of valence, arousal, and dominance as dependent variables. It is worth noting that even though the number of males and females included in the study are not equivalent (each set was rated by 100 subjects, with a male:female ratio that approached 1:3 in each set), the splithalf analyses conducted on the male and female ratings in each of the 20 stimulus sets revealed that the SpearmanBrown reliability coefficient were satisfactorily distributed across the stimulus sets for each of the three affective

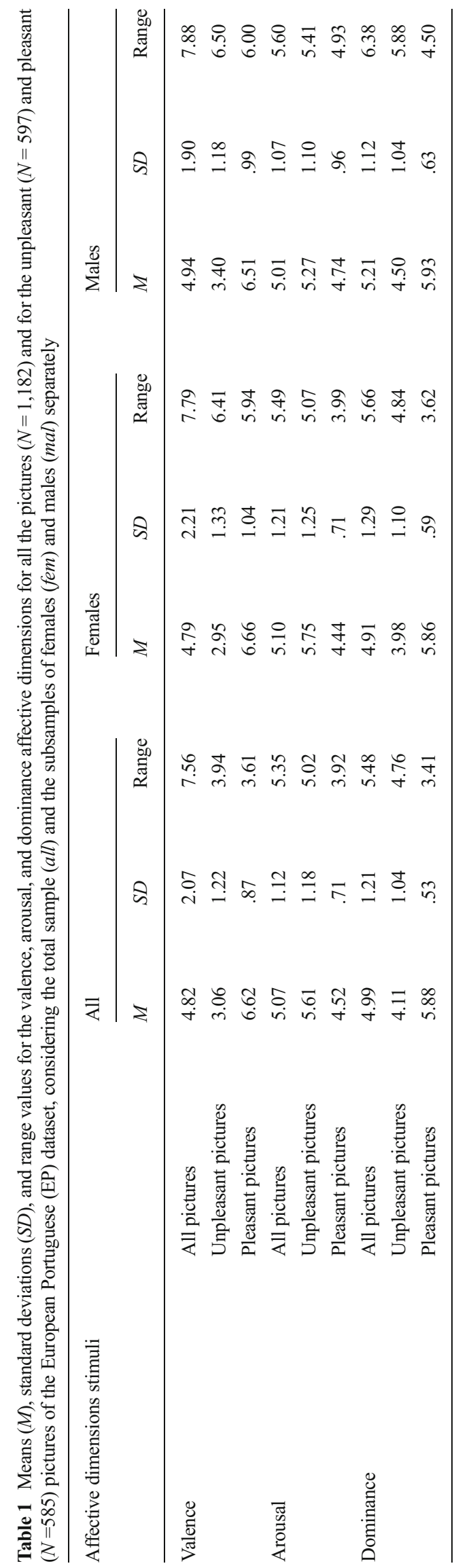


dimensions, ranging in the male subsample from .65 to .98 in the valence affective dimension, from .72 to .97 in the arousal dimension and from .62 to .98 in the dominance affective dimension (in the female subsample they ranged from .67 to .92 in the valence affective dimension, from .71 to .97 in the arousal dimension and from .66 to .95 in the dominance dimension). Therefore, in spite of the discrepancy in the number of males and females in the adaptation of the IAPS to EP, these results confirm the reliability of IAPS ratings when considering the male (and female) subsamples separately.

The MANOVA analysis showed a main effect of picture valence on the affective ratings of valence, $F(1,2360)=$ $5222.28, p<.001, \eta^{2}=.69$, arousal, $F(1,2360)=474.46, p$ $<.001, \eta^{2}=.17$, and dominance, $F(1,2360)=2121.91, p<$ $.001, \eta^{2}=.47$. It also showed a significant main effect of sex on the affective dimensions of valence, $F(1,2360)=10.03, p$ $<.01, \eta^{2}=.004$, arousal, $F(1,2360)=4.39, p<.05, \eta^{2}=.002$, and dominance, $F(1,2360)=67.703, p<.001, \eta^{2}=.028$. A significant interaction between sex and picture valence for the valence, $F(1,2360)=41.09, p<.001, \eta^{2}=.017$, arousal, $F(1$, $2360)=83.84, p<.001, \eta^{2}=.034$, and dominance, $F(1,2360)$ $=37.47, p<.001, \eta^{2}=.016$, affective dimensions was also observed.

The post-hoc Scheffé contrasts for the main effect of picture valence showed, as expected, that pleasant pictures were rated more positively than unpleasant pictures $(p<.001)$. Also, in line with the abovementioned findings, unpleasant pictures were rated as more arousing than the pleasant ones $(p<.001)$, and additionally with lower levels of dominance $(p<.001)$. These findings confirm the reported asymmetry between valence and arousal for pleasant and unpleasant stimuli (e.g., Bradley \& Lang, 2000; Bradley et al., 2001a; Soares et al., 2012, 2013), as well as the fact that unpleasant stimuli tend to elicit subjective feelings of lower control (see LeDoux, 1996).

Furthermore, results showed that while Portuguese males rated IAPS stimuli with higher levels of dominance $(p<.001)$ and valence $(p<.01)$ than females, Portuguese females rated IAPS stimuli with higher levels of arousal $(p<.05)$ than males. These results confirm a pattern already observed in previous studies with IAPS pictures (e.g., Bradley et al., 2001b; Dufey et al., 2011; Moltó et al., 1999; Vila et al., 2001), as well as the results observed in the adaptation of the ANEW and IADS-2 sets for EP (see Soares, 2012, 2013 for details). These differences are further observed when the two motivational systems were taken into consideration in the analysis. Figure 4 illustrates the distribution of the EP IAPS ratings in the bi-dimensional affective space of valence and arousal for the subsamples of females and males separately.

As shown in Fig. 4, the interaction between sex and picture valence for the arousal affective dimension reveals that in the appetitive motivational system males rated pleasant pictures as more arousing than females $(p<.001)-$ as revealed by the concentration of higher ratings of males than females in the upper right quadrant of the chart. In the defensive motivational system females rated unpleasant pictures as more arousing than males $(p<.001)$ - as revealed by the concentration of lower ratings of females than males in the lower left quadrant of the chart. This finding shows that Portuguese females reveal a stronger reactivity toward unpleasant IAPS pictures (i.e., a negativity bias), whereas Portuguese males showed a stronger emotional reactivity toward pleasant IAPS pictures (i.e., a positivity bias). This asymmetry is not entirely new and is consistent with which has been observed in other studies (e.g., Bradley \& Lang, 2007b; Bradley et al., 2001b). For example, Bradley and Lang (2007b) found that approximately $40 \%$ of males showed a positivity bias (and only $15 \%$ a negativity bias), while $30 \%$ of females showed a negativity bias (and only $16 \%$ a positivity bias).

Of note, a detailed analysis of the pleasant pictures rated as more arousing by Portuguese males showed that these ratings are essentially related to erotic stimuli (e.g., pictures 4300 , 4008,4142 , or $4003 ; M_{\text {valence }}=8.53,8.33,8.23,8.02$, respectively; $M_{\text {arousal }}=7.13,7.29,7.23,7.12$, respectively), whereas in the case of Portuguese females the pleasant pictures that were rated as more arousing are related to categories as distinct as "IceCream" (e.g., pictures 7330 or $7340, M_{\text {valence }}$ $=8.28,8.24 ; M_{\text {arousal }}=6.16,6.08$, respectively), "Concert" (picture $7499, M_{\text {valence }}=7.90 ; M_{\text {arousal }}=6.80$ ), "Bungee" (picture $8179 M_{\text {valence }}=7.43 ; M_{\text {arousal }}=6.37$ ), "CliffDivers" (e.g., pictures 8180 or $8178 M_{\text {valence }}=7.18,6.83 ; M_{\text {arousal }}=$ $6.78,6.36$, respectively) or "Gold" $\left(8500, M_{\text {valence }}=7.72\right.$; $\left.M_{\text {arousal }}=6.02\right)$. Still, the arousal scores for those pictures in women are lower than the arousal scores observed for the erotic stimuli in men.

In line with our hypothesis, this finding confirms the idea that erotic stimuli have a 'special' status within the category of positively valenced stimuli in men, and also that the mechanisms associated with the processing of erotic stimuli seem to be different for males and females (e.g., Bradley et al., 2001b; Karama et. al., 2002; Yi et al., 2006; Yu et al., 2012). Indeed, even though Portuguese males rated erotic stimuli (e.g., pictures $4300,4008,4142$, or 4003$)$ as very positive $\left(M_{\text {valence }}=\right.$ $8.53,8.33,8.23,8.02$, respectively) and highly arousing $\left(M_{\text {arousal }}=7.13,7.29,7.23,7.12\right.$, respectively $)$, Portuguese females rated the same stimuli not only with lower levels of arousal $\left(M_{\text {arousal }}=3.92,4.16,3.68,4.05\right.$, respectively $)$, but also with considerably lower levels of valence $\left(M_{\text {valence }}=\right.$ $4.05,4.79,3.32,4.93$, respectively). Therefore, future extensions of the IAPS set should consider not only an increment of the number of high-arousing pleasant stimuli as suggested earlier, but importantly that these new pictures be sensitive both to men and women characteristics.

In the defensive motivational system, the sex differences were less pronounced. Nonetheless, it is important to note that, even though the content of most of the unpleasant 


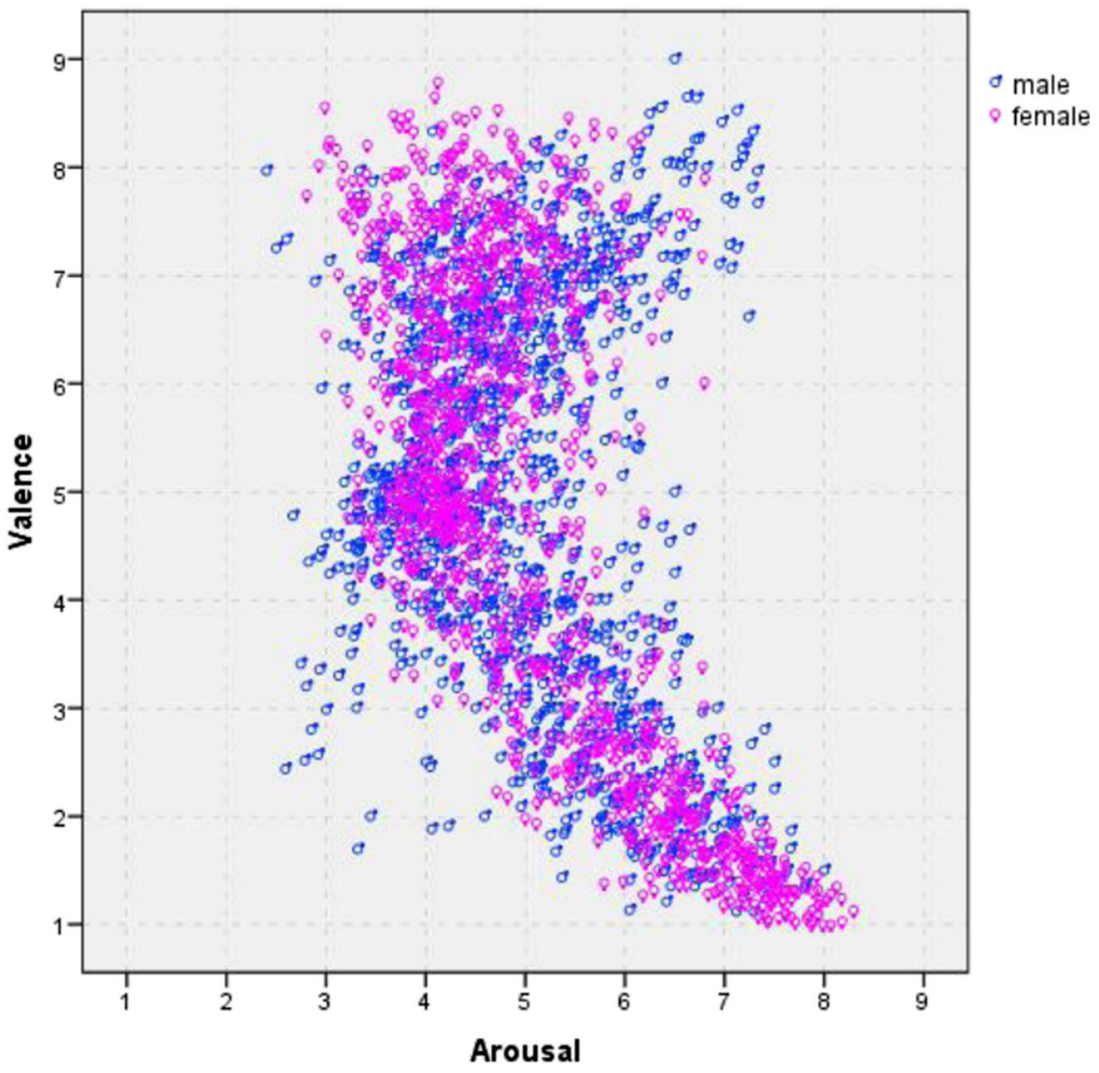

Fig. 4 Distribution of the mean values for the valence and arousal affective dimensions in the subsamples of females and males separately in the European Portuguese (EP) standardization of the IAPS

pictures was similar for males and females, including essentially pictures of mutilated and injured people (e.g., 3001, 3053, 3101) (females: $M_{\text {valence }}=1.13,1.00,1.03$, respectively; males: $M_{\text {valence }}=1.50,1.50,1.57$, respectively), Portuguese women rated those pictures as more arousing $(p<.001)$ than Portuguese men (females: $M_{\text {arousal }}=8.29,7.99,8.13,8.18$, respectively; males: $M_{\text {arousal }}=8.00,7.63,7.44,7.32$ ).

Additionally, the interaction between sex and picture's valence in the valence affective dimension showed that Portuguese females use more extreme scores than Portuguese males, when rating IAPS stimuli both in the defensive and in the appetitive systems, i.e., they rated unpleasant pictures not only as more negative $(p<.001)$, but also pleasant pictures as more positive $(p<.05)$ than males. This finding was only observed when the two motivational systems were considered in the analysis (bear in mind that when the two motivational systems are not considered, it was found that males rated IAPS stimuli with higher valence scores than females - as the main effect of valence aforementioned demonstrated). These findings support our hypothesis and highlight the need to consider the appetitive and defensive motivational systems separately if we want to obtain a more complex and enriched picture of how sex affects IAPS ratings. As expected, Portuguese females show a stronger emotional reactivity towards IAPS stimuli than Portuguese males, which should be taken into consideration when conducting affective research in Portugal. Finally, considering the dominance dimension, the interaction between sex and picture's valence indicated that, although men showed higher levels of dominance than women, these differences only reached statistical significance in the defensive motivational system, i.e., for the unpleasant IAPS pictures $(p<.001)$.

Taken together, the results of the IAPS adaptation to EP indicate that, even though IAPS stimuli are effective in inducing a range of affective reactions that are properly distributed in the bi-dimensional affective space of valence and arousal (as shown by the boomerang-shaped distribution), there are important differences in the way Portuguese males and females react to IAPS stimuli, and these differences cannot be neglected when planning research on affective picture processing with EP participants. Of note, these differences were more pronounced than those previously observed in the adaptation of the ANEW (Soares et al., 2012) and the IADS-2 (Soares et al., 2013) sets for EP. In the ANEW standardization, the main effect of sex was only significant for the arousal dimension (see Soares et al., 2012 for details), and in the IADS-2 standardization, sex differences were only observed for the arousal dimension when the defensive and appetitive motivational systems were taken into consideration (see Soares et al., 2013 for details). The larger quantity and 
complexity of the information conveyed by pictures relative to words or sounds may contribute to enhancing differences in the way Portuguese males and females affectively react to the IAPS stimuli. These differences are also in line with the findings of recent studies using both behavioral and psychophysiological measures of emotions (e.g., Bradley et al., 2001a; Kemp et al., 2004; Lithari et al., 2010; Yi et al., 2006; Wrase et al., 2003) and highlights differences in the processing of different types of affective stimuli (e.g., De Houwer \& Hermans, 1994; Hinojosa et al., 2009). Therefore, the observed sex differences strongly recommend the use of male and/or female IAPS norms when conducting research on affective processing with EP participants.

\section{Crosscultural rating of IAPS stimuli}

In order to explore crosscultural differences in the way Portuguese subjects and subjects from different countries and cultures rated IAPS pictures, we compared, as mentioned before, the EP ratings with the ratings of other IAPS standardizations to which normative values we had access: USA (Lang et al., 2008), SP (Moltó et al., 1999; Vila et al., 2001), BR (Lasaitis et al., 2008; Ribeiro et al., 2005), BG (Verschuere et al., 2001), $\mathrm{CH}_{1}$ (Dufey et al., 2011), $\mathrm{CH}_{2}$ (Silva, 2011), IND (Lohani et al., 2013), and BH (Drače et al., 2013) standardizations. It is worth noting that although all the cited IAPS adaptations followed Lang et al.'s (1995-2008) original procedure in terms of data collection, they do not explicitly mention the size of the IAPS pictures used. Picture size is an important variable to control for, as it has been demonstrated that it plays an important role in affective ratings. For instance, in a study that examined the effects of picture size on emotional reactions, Codispoti and De Cesarei (2007) found that picture size (small, medium, and large) modulates affective ratings. Arousal ratings decreased significantly with smaller picture size, while in the valence scale the effect of size varied as a function of stimulus content (i.e., pleasant and neutral pictures decreased in pleasantness as picture size diminished, whereas unpleasant pictures decreased in pleasantness as picture size became larger). Nevertheless, since all these normative studies tried to replicate the original procedure adopted by Lang et al. and did not make any reference to size manipulation, in our crosscultural analysis we relied on the assumption that all these studies, as ours, presented the IAPS pictures in their original size.

Figure 5 shows the distribution of the mean values of valence and arousal for the common pictures between the EP and the USA IAPS set $(N=1,182)$, the EP and the SP IAPS set $(N=820)$, the EP and the BR IAPS set $(N=916)$, the EP and the BG IAPS set $(N=60)$, the EP and the IND IAPS set $(N=100)$, and the EP and the BH IAPS set $(N=60)$. For the Chilean standardization, Fig. 5 presents only the results of the common pictures between EP and the Dufey et al. (2011)
Fig. 5 Distribution of the mean values for the valence and arousal affective dimensions in the total sample in the European Portuguese (EP) American (USA; Lang et al., 2008), Spanish (SP; Moltó et al., 1999; Vila et al., 2001), Belgian (BG; Verschuere et al., 2001), Brazilian (BR; Lasaitis et al., 2008; Ribeiro et al., 2005), Chilean $\left(\mathrm{CH}_{1}\right.$; Dufey et al., 2011), Indian (IND; Lohani et al., 2013), and BosniaHerzegovinian (BH; Drače et al., 2013) standardizations of the International Affective Picture System (IAPS)

study $(N=188)\left(\mathrm{CH}_{1}\right)$ because, as mentioned above, Silva (2011) only provided normative values for the subsamples of females and males separately (the comparison with the Silva et al. work $\left[\mathrm{CH}_{2}\right]$ was considered in the following analysis and for 118 common pictures). ${ }^{4}$

The distribution of the valence and arousal scores in the eight IAPS standardizations (cf. Fig. 5), indicates a great overlap, with all revealing the expected boomerang-shape . $^{5}$ In all the eight IAPS standardizations, the quadratic relationship between valence and arousal was higher and captured more variance (USA: $r=.33, R^{2}=.11, p<.001$; SP: $r=.55, R^{2}$ $=.30, p<.001 ; \mathrm{BR}: r=.84, R^{2}=.71, p<.001 ; \mathrm{BG}: r=.30, R^{2}$ $=.09, p=.069 ; \mathrm{CH}_{1}: r=.69, R^{2}=.48, p<.001 ; \mathrm{IND}: r=.12$, $R^{2}=.01, p=.507$; BH: $r=.63, R^{2}=.38, p<.001$; EP: $r=.70$, $\left.R^{2}=.49, p<.001\right)$ than their corresponding pairwise linear correlations (USA: $r=.29, R^{2}=.08, p<.001$; SP: $r=.52, R^{2}=$ $.27, p<.001$; BR: $r=.83, R^{2}=.69, p<.001 ; \mathrm{BG}: r=.19, R^{2}=$ $.02, p=.137 ; \mathrm{CH}_{1}: r=.62, R^{2}=.38, p<.001$; IND: $r=.12, R^{2}$ $=.01, p=.249$; $\mathrm{BH}: r=.49, R^{2}=.22, p<.001 ; \mathrm{EP}: r=.68, R^{2}$ $=.46, p<.001)$. Nonetheless, it is important to note that in the BG standardization, the quadratic correlation is only marginally significant (and the linear correlation is not significant) and, in the IND standardization, both correlations were nonsignificant. As pointed out by Lohani et al. (2013), this result might reveal important sociocultural specificities in the way Indian people react affectively to IAPS stimuli when compared with subjects from Western cultures. For example, contrary to what is observed in all the other IAPS standardizations, in the IND standardization the relationship between

\footnotetext{
${ }^{4}$ It is worth noting that since USA and BR standardizations provide normative values for the 12 pictures $(1230,1590,1610,1640,1670$, $2210,3000,3010,4520,6200,6571$, and 9090) and the SP standardization for ten pictures $(1230,1590,1610,1640,1670,2210,3000,3010$, 4220 , and 4520) twice (as they integrated two different sets), we included the normative values of those pictures only once in the analysis in order to avoid the duplication of values for the same IAPS stimuli. Nonetheless, it is worth noting that the values considered for each of those pictures were obtained from the same set. For instance, the normative values considered for picture 1230 were obtained from set 2 in any of the IAPS standardizations under analysis.

${ }^{5}$ Even though the authors of each of the IAPS standardizations provided values for the linear and/or the quadratic correlations between valence and arousal, the values reported here were re-calculated since we considered only the values of the pictures that were common between the EP and each of the other IAPS standardizations, and excluded, as mentioned before, the duplications of data for pictures that were associated with more than one set.
} 

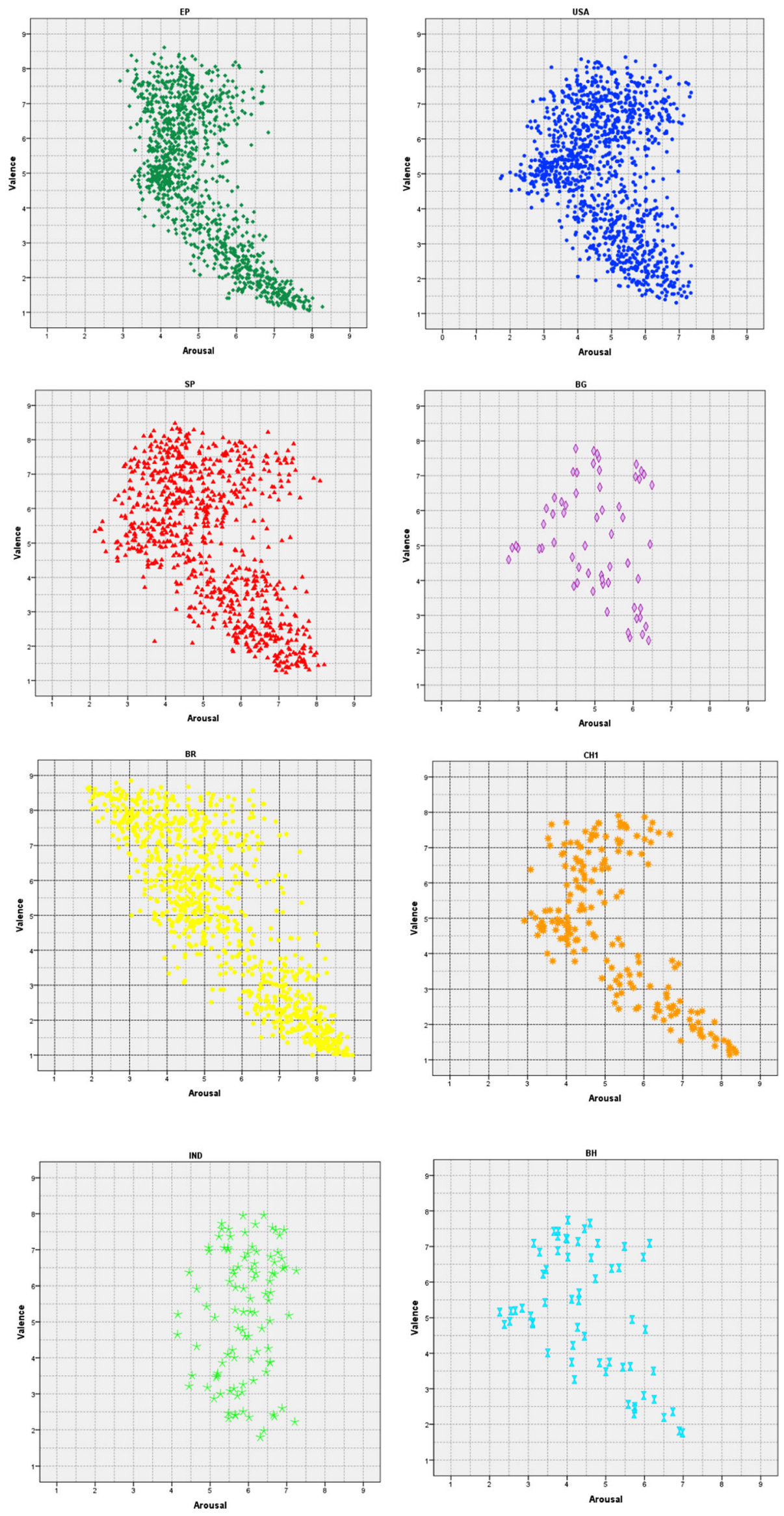
valence and arousal was stronger for the appetitive than for the defensive motivational system (for details see Lohani et al., 2013). This result may suggest that, although differences in the way subjects rated IAPS pictures may be subtler in individuals from Western cultures, these differences may become more evident when we compare individuals from more differentiated cultures, as for example Indian and EP people. Exploring the reasons for these differences is beyond the scope of this paper. Still, they seem to support the idea advanced by Mesquita and Walker (2003) that culture shapes the way individuals perceive, feel, and express emotions, which should be confirmed in future studies. Collecting data from the same affective dimensions and the same type of stimuli in individuals from different countries and cultures might be a promising line of research.

The results of the distribution of the IAPS ratings in the bidimensional affective space showed that in EP the quadratic correlation between valence and arousal is higher $(r=.70)$ and captures more variance $(49 \%)$ than in any other IAPS standardizations, with the exception of the BR standardization $(r=$ $.83, R^{2}=.71$ ). This indicates that, in an EP context (as in Brazil), the valence and arousal affective dimensions greatly account for the variability of results observed in the way participants rated IAPS pictures.

Table 2 presents the results for Pearson correlations between $\mathrm{EP}$ and the USA, SP, BR, BG, $\mathrm{CH}_{1}, \mathrm{CH}_{2}$, IND, and $\mathrm{BH}$ IAPS standardizations for the valence, arousal, and dominance affective dimensions considering the total sample, and for the subsamples of females and males separately. For the reasons previously mentioned, Table 2 presents the results of the correlation analysis between $\mathrm{EP}$ and the $\mathrm{BG}, \mathrm{CH}_{1}, \mathrm{IND}$ and BH IAPS standardizations only for the total sample (all) of subjects and, in the case of the $\mathrm{CH}_{1}$ standardization, only for the valence and arousal dimensions. In the case of the $\mathrm{CH}_{2}$ standardization, the results in Table 2 are only presented for males and females separately and for the valence and arousal affective dimensions.

As presented in Table 2, all correlations were positive and highly significant $(p<.001$ in all comparisons), except for the correlations between EP and IND in the arousal and dominance affective dimensions. These findings replicate the observation of Lohani et al. (2013) when comparing the Indian and USA samples. As was observed with the USA subjects, EP and IND subjects were similar in valence ratings but differed significantly in arousal and dominance ratings: Indian participants showed higher levels of arousal and dominance than Portuguese participants. In the Indian sample, the minimum rating of arousal was 4.15, while in the Portuguese sample it was 3.32. Considering the dominance dimension, the minimum rating was 3.85 in the Indian sample, and 2.28 in the Portuguese sample. As suggested by Lohani et al. (2013), the reasons underlying these differences are not entirely clear but may arise from important cultural differences in the way Indian subjects and subjects from

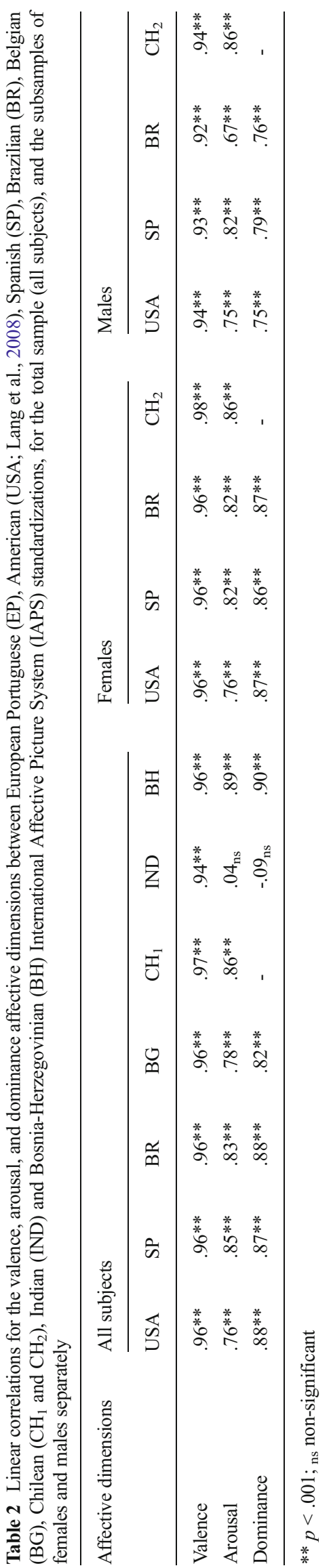


Western cultures (as Portuguese participants) might interpret the specific content of the arousal and dominance constructs. Furthermore, the high correlations observed in the affective dimension of valence (.94) might suggest that the way individuals evaluate the positive or negative information conveyed by the stimuli is determined more by biological processes than by social and cultural responses. As Bradley and collaborators (Bradley \& Lang, 1994; Bradley et al., 2001a) state, the evaluation of stimuli as positive or negative activates the defensive or the appetitive motivational systems, respectively, which in turn elicits responses of withdrawal or approach that represent, from an evolutionary point of view, an important survival mechanism (see also Sakaki, Niki, \& Mather, 2012). The primary assessment of stimuli as positive or negative may therefore be more automatic than the assessment of other affective dimensions, and hence less prone to social and cultural influences. Additionally, the reduced number of IAPS pictures $(N=100)$ and of participants $(N=80)$ in the Indian IAPS standardization, as well as the fact that those pictures depicted sports and animals that are common in Western cultures but not in Indian culture, as pointed out by Lohani et al. (2013), may also play a role in these results. More research is thus needed in order to better explain these results.

Nevertheless, it is important to highlight that in all the other standardizations, the correlations were high and statistically significant both when the global sample (with correlation values ranging from .76 to .97) and the subsamples of females (with correlation values ranging from .76 to .98) and males (with correlations value ranging from .75 to .94) were considered separately. Moreover, the comparison of the correlation values across IAPS standardizations revealed great stability in each of the affective dimensions considered, particularly in the valence dimension (with correlation values ranging between .92 to .98) which may be due, as mentioned above, to the fact that the pleasantness of the stimuli might be assessed more automatically than the assessment of other affective dimensions. Nevertheless, the results of the correlation analyses showed that IAPS stimuli evoke similar emotional responses in individuals from different countries and cultures, which strongly supports the crosscultural validity of this set, at least in Western cultures.

Table 3 presents means, standard deviations and range scores in the three affective dimensions of valence, arousal, and dominance for the global sample (all) and for the subsamples of females and males separately in each of the IAPS standardizations under analysis. For reasons previously mentioned, in the $\mathrm{BG}, \mathrm{IND}, \mathrm{BH}$, and $\mathrm{CH}_{1}$ standardizations, Table 3 presents results of valence, arousal, and dominance only for the global sample (and in the $\mathrm{CH}_{1}$ only for the valence and arousal affective dimensions), and in the case of the $\mathrm{CH}_{2}$ standardization only for males and females separately for the valence and arousal affective dimensions.
In order to further explore the crosscultural differences in the way Portuguese subjects and subjects from other countries and cultures rated IAPS pictures, we conducted a MANOVA with IAPS standardization (EP, USA, SP, BR, BG, $\mathrm{CH}_{1}, \mathrm{CH}_{2}$, IND, and $\mathrm{BH}$ ) and sample type (global sample, female subsample, male subsample) as between-subject factors and the affective dimensions of valence, arousal, and dominance as dependent variables. Since $\mathrm{CH}_{1}$ and $\mathrm{CH}_{2}$ standardizations only provide valence and arousal ratings, we ran two distinct MANOVAs. The first was conducted for the valence and arousal affective dimensions considering all the standardizations under analysis (EP, USA, SP, BR, BG, $\mathrm{CH}_{1}, \mathrm{CH}_{2}, \mathrm{IND}$, and $\mathrm{BH}$ ), and the second MANOVA only for the dominance dimension considering the standardizations for which the dominance ratings were available (EP, USA, SP, BR, BG, IND, and $\mathrm{BH}$ ).

The results of the two MANOVAs showed a main effect of IAPS standardization in the affective dimensions of valence, $F(8,12926)=4.45, p<.001, \eta^{2}=.003$, arousal, $F(8,12926)=$ $62.69, p<.001, \eta^{2}=.037$, and dominance, $F(6,12505)=$ $24.51, p<.001, \eta^{2}=.012$, as well as a main effect of sample type in the affective dimensions of valence, $F(2,12926)=$ 7.07, $p<.001, \eta^{2}=.001$, arousal, $F(2,12926)=11.41, p<$ $.001 . \eta^{2}=.002$, and dominance, $F(2,12505)=58.95, p<$ $.001 . \eta^{2}=.009$. No interaction reached statistical significance.

The post-hoc Scheffé contrasts for the main effect of the IAPS standardization with the Bonferroni correction for multiple comparisons, revealed that EP participants rated IAPS pictures with lower levels of valence than USA $(p<.001)$, the SP $(p<.001)$, and BR $(p<.001)$ subjects. In the arousal dimension, EP participants rated IAPS pictures with higher levels of arousal than USA $(p<.001)$ and $\mathrm{CH}_{2}(p<.001)$ subjects, but with lower levels of arousal than SP $(p<.01)$, BR $(p<.001)$ and IND $(p<.001)$ subjects. Finally, in the dominance dimension, EP subjects rated IAPS pictures with higher levels of control than the SP $(p<.001)$ subjects, but with lower levels than USA $(p<.001)$ and BH $(p<.01)$ subjects. Concerning the main effect of sex, results showed that irrespective of the IAPS standardization, males rated IAPS pictures with higher levels of valence $(p<.001)$ and dominance $(p<.001)$ than females. This result is also observed when male ratings were compared with the ratings obtained in the global sample of subjects, both for the valence $(p<.01)$ and for the dominance $(p<$.001) affective dimensions. Furthermore, and regardless of the IAPS standardization, females showed higher levels of arousal than males $(p<.001)$.

Together these findings revealed that, in spite of similarities in the way people from different countries and cultures react to IAPS stimuli, there are some crosscultural differences that should not be neglected. Even though caution is needed when interpreting the comparison with the $\mathrm{BG}, \mathrm{CH}$, IND, and $\mathrm{BH}$ standardizations due to the reduced number of stimuli included in those studies, comparison with the other standardizations 


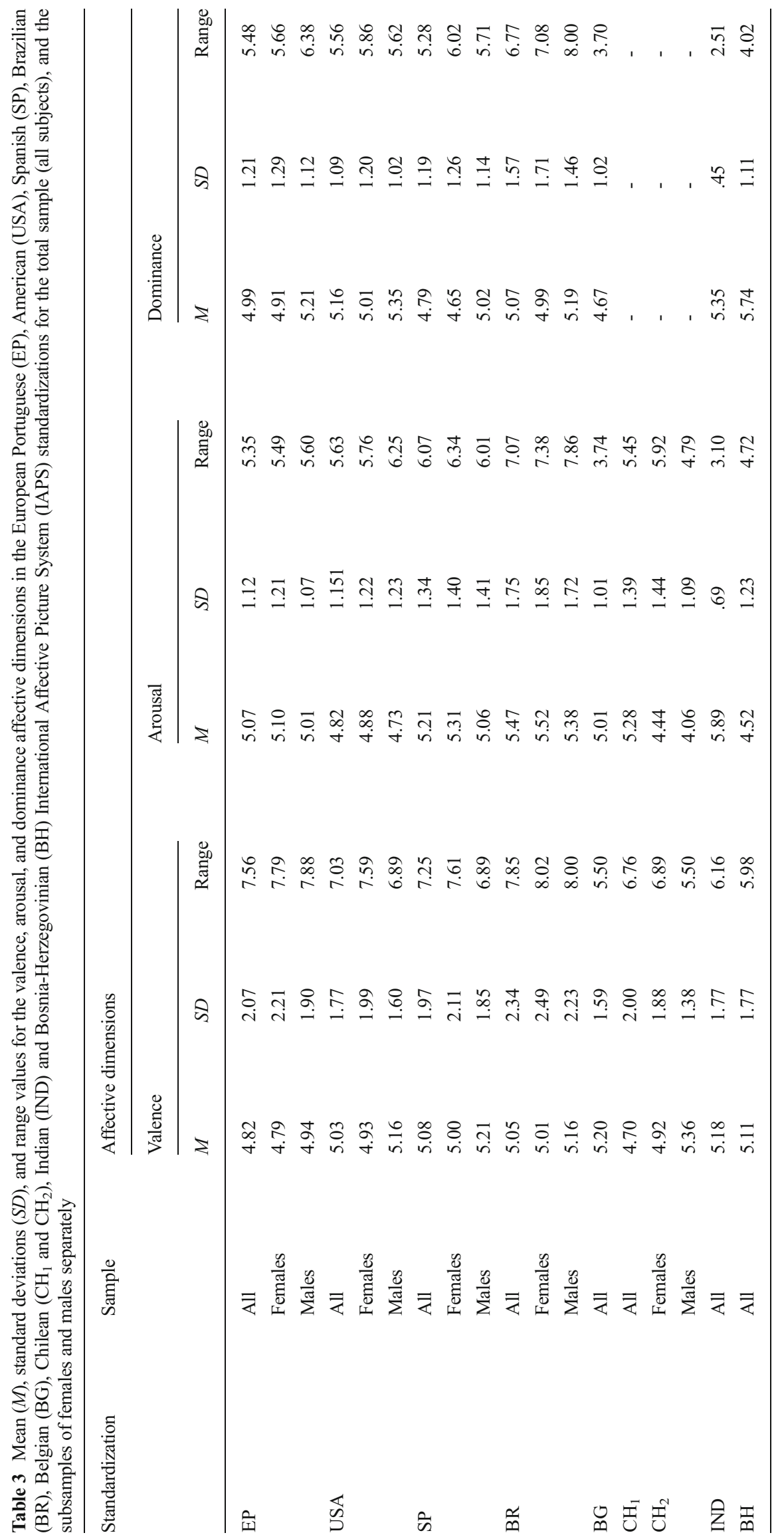


revealed that Portuguese subjects rated IAPS stimuli with the lowest levels of valence (although the differences only reached statistical significance when EP individuals were compared to USA, SP, and BR individuals). Moreover, Portuguese individuals rated IAPS stimuli with lower levels of arousal than SP and BR subjects (but higher than USA and $\mathrm{CH}$ subjects), and also with lower levels of dominance than USA, BR, and BH subjects (but higher than SP subjects). As mentioned above, exploring the reasons for these differences is beyond the scope of this paper and should be further explored in future studies. Nonetheless, it is worth noting that the crosscultural differences observed in the way Portuguese subjects and subjects from other countries and cultures react to IAPS stimuli are consistent with those previously observed in the adaptation of the ANEW (Soares et al., 2012) and IADS sets for EP (Soares et al., 2013). These findings suggest that Portuguese individuals are less emotionally reactive than individuals from other countries and cultures to affective stimuli, at least for those included in the CSEA sets. These differences strongly recommend the use of the normative values presented here when planning and conducting research with Portuguese individuals. The way people experience and express emotions seems to result from a complex interplay of social and cultural responses, underlying biological processes as well as the type of information conveyed by the stimuli. Future research should further explore the dynamics between those factors in shaping the emotional experience of individuals coming from different countries and cultures.

\section{Conclusion}

The aim of this study was to collect norms of valence, arousal, and dominance of the last version of the IAPS set (Lang et al., 2008) for EP. The use of standardized stimuli is needed to effectively support research on affective processing. The results of the EP adaptation of the IAPS presented here indicate that the IAPS is a reliable and useful tool for the study of attention and emotion processing with Portuguese participants. Furthermore, the use of this set allows the comparability of results with other international studies using the same stimuli. This work completes the adaptation of the three sets of affective stimuli created by the CSEA (ANEW; Bradley, \& Lang, 1999a; IADS-2; Bradley, \& Lang, 1999b, 2007a) for EP (Soares et al., 2012, 2013, respectively), which represents an important contribution for the scientific community, since it provides valuable research tools to promote the development of Portuguese research on affective processing, using both unimodal and multimodal paradigms.

Similar to those previously observed in the EP adaptation of the ANEW (Soares et al. 2012) and the IADS-2 (Soares et al., 2013), the results reported here confirm that in an EP context the responses to IAPS pictures are properly distributed in the two motivational systems (defensive and appetitive) that regulate emotion expression (Bradley \& Lang, 2000; Bradley et al., 2001a). Moreover, in line with recent evidence (e.g., Bradley et al., 2001b; Karama et. al., 2012; Kemp et al., 2004; Lithari et al., 2010; Yi et al., 2006; Yu et al., 2012; Wrase et al., 2003) our findings revealed that Portuguese males and females react differently to IAPS pictures and that these sex differences should be taken into account when planning and conducting emotion research with Portuguese individuals.

Finally, the crosscultural analyses conducted with the eight standardizations for whose normative values we had access to showed that, even though IAPS stimuli elicit affective reactions that are similar in individuals coming from different countries and cultures (which supports its crosscultural validity and allows the comparability of results across international studies), there are sociocultural specificities that should be acknowledged. In line with the previous EP findings with emotionally evocative stimuli (Soares et al., 2012, 2013), Portuguese subjects revealed a lower emotional reactivity towards IAPS pictures than USA, SP, and BR subjects. Therefore, it is crucial that attention and emotion research conducted with Portuguese samples uses the normative values provided in this work. These are freely available for research purposes at http://brm.psychonomic-journals.org/content/ supplemental or at http://p-pal.di.uminho.pt/about/databases.

Acknowledgments Ana Paula Soares is also Director of the Human Cognition Laboratory (HCL) of the School of Psychology, University of Minho, Portugal, and the Main Researcher of the Research Group of Psycholinguistics (RGP) hosted at the HCL. This work is part of the research project "Procura Palavras (P-Pal): A software program for deriving objective and subjective psycholinguistic indices for European Portuguese words" (PTDC/PSI-PCO/104679/2008), funded by FCT (Fundação para a Ciência e Tecnologia), and FEDER (Fundo Europeu de Desenvolvimento Regional) through the European programs QREN (Quadro de Referência Estratégico Nacional), and COMPETE (Programa Operacional Factores de Competitividade). It was also supported by Grant PTDC/PSI-PCL/116626/2010 from FCT and FEDER (Fundo Europeu de Desenvolvimento Regional) through the European programs QREN (Quadro de Referência Estratégico Nacional), and COMPETE (Programa Operacional Factores de Competitividade), awarded to A.P.P.

\section{References}

Barke, A., Stahl, J., \& Kröner-Herwig, B. (2012). Identifying a subset of fear-evoking pictures from the IAPS on the basis of dimensional and categorical ratings for a German sample. Journal of Behavior Therapy and Experimental Psychiatry, 43, 565-572.

Beaupre, M. G., Cheung, N., \& Hess, U. (2000). The Montreal set of facial displays of emotion. Quebec, Canada: Montreal.

Belin, P., Fillion-Bilodeau, S., \& Gosselin, F. (2008). The Montreal affective voices: A validated set of nonverbal affect bursts for research on auditory affective processing. Behavior Research Methods, 40, 531-539. 
Bradley, M. M., \& Lang, P. J. (1994). Measuring emotion: The self assessment Manikin and the semantic differential. Journal of Behavior Therapy and Experimental Psychiatry, 25, 49-59.

Bradley, M. M., \& Lang, P. J. (1999a). Affective norms for English words $(A N E W)$ : Instruction manual and affective ratings. Gainesville, FL: Center for Research in Psychophysiology. University of Florida.

Bradley, M. M., \& Lang, P. J. (1999b). International affective digitized sounds (IADS): Stimuli, instruction manual and affective ratings. Gainesville, FL: The Center for Research in Psychophysiology. University of Florida.

Bradley, M. M., \& Lang, P. J. (2000). Measuring emotion: Behavior, feeling and physiology. In R. Lane \& L. Nadel (Eds.), Cognitive neuroscience of emotion (pp. 242-276). New York: Oxford University Press.

Bradley, M. M., \& Lang, P. J. (2007a). International affective digitized sounds (2nd Edition; IADS-2): Stimuli, instruction manual and affective ratings. Gainesville, FL: The Center for Research in Psychophysiology. University of Florida.

Bradley, M., \& Lang, P. J. (2007b). The international affective picture system (IAPS) in the study of emotion and attention. In J. A. Coan \& J. J. B. Allen (Eds.), Handbook of emotion elicitation and assessment (pp. 29-46). New York: Oxford University Press.

Bradley, M. M., Cuthbert, B. N., \& Lang, P. J. (1999). Affect and the startle reflex. In M. E. Dawson, A. Schell, \& A. Boehmelt (Eds.), Startle modification: Implications for neuroscience, cognitive science and clinical science (pp. 157-183). Stanford, CA: Cambridge.

Bradley, M. M., Codispoti, M., Sabatinelli, D., \& Lang, P. J. (2001a). Emotion and motivation. I: Defensive and appetitive reactions in picture processing. Emotion, 1, 276-298.

Bradley, M. M., Codispoti, M., Sabatinelli, D., \& Lang, P. J. (2001b). Emotion and motivation. II: Sex differences in picture processing. Emotion, 1, 300-319.

Bradley, M. M., Greenwald, M. K., Petry, M. C., \& Lang, P. J. (1992). Remembering pictures: Pleasure and arousal in memory. Journal of Experimental Psychology: Learning, Memory, and Cognition, 18, 379-390.

Bradley, M. M., Hamby, S., L w, A., \& Lang, P. J. (2007). Brain potentials in perception: Picture complexity and emotional arousal. Psychophysiology, 44, 364-373.

Bradley, M. M., Miccoli, L., Escrig, M. A., \& Lang, P. J. (2008). The pupil as a measure of emotional arousal and autonomic activation. Psychophysiology, 45, 602-607.

Briesemeister, B. B., Kuchinke, L., \& Jacobs, A. M. (2011). Discrete emotion norms for nouns: Berlin Affective Word List (DENNBAWL). Behavior Research Methods, 43, 441-448.

Calvo, M. G., \& Avero, P. (2009). Reaction time normative data for the IAPS as a function of display time, gender, and picture content. Behavior Research Methods, 41, 184-191.

Carretié, L., Mercado, F., Tapia, M., \& Hinojosa, J. A. (2001). Emotion, attention, and the 'negativity bias', studied through event-related potentials. International Journal of Psychophysiology, 41, 75-85.

Castillo-Parra, G., De Jesús, A. I., Ostrosky-Solís, J., \& Ostrosky-Solís, F. (2002). Valencia, Activación y Tiempos de Reacción ante Estímulos Visuales con Contenido Emocional: Un Estudio en Población Mexicana [Valence, arousal and reaction times for visual stimuli with emotional content: a study with a Mexican sample]. Revista Mexicana de Psicología, 19, 167e176.

Codispoti, M., \& De Cesarei, A. (2007). Arousal and attention: Picture size and emotional reactions. Psychophysiology, 44, 680-686.

Codispoti, M., Ferrari, V., \& Bradley, M. M. (2006). Repetitive picture processing: Autonomic and cortical correlates. Brain Research, 1068, 213-220.

Comesaña, M., Soares, A. P., Perea, M., Piñeiro, A., Fraga, I., \& Pinheiro, A. P. (2013). ERP correlates of masked affective priming with emoticons and words. Computers in Human Behavior, 29, 588-595.
Cuthbert, B. N., Schupp, H. T., Bradley, M. M., Birbaumer, N., \& Lang, P. J. (2000). Brain potentials in affective picture processing: Covariation with autonomic arousal and affective report. Biological Psychology, 52, 95-111.

Dan-Glauser, E. S., \& Scherer, K. R. (2011). The Geneva affective picture database (GAPED): A new 730-picture database focusing on valence and normative significance. Behavior Research Methods, 43, 468-477.

Davis, W. J., Rahman, M. A., Smith, L. J., Burns, A., Senecal, L., McArthur, D., \& Wagner, W. (1995). Properties of human affect induced by static color slides (IAPS): Dimensional, categorical and electromyographic analysis. Biological Psychology, 41, 229-253.

De Houwer, J., \& Hermans, D. (1994). Differences in the affective processing of words and pictures. Cognition and Emotion, 8(1), 120.

Déak, A., Csenki, L., \& Révész, G. (2010). Hungarian ratings for the international affective picture system (IAPS): A cross-cultural comparison. Empirical Text and Culture Research, 4, 90-101.

Drače, S., Efendić, E., Kusturica, M., \& Landžo, L. (2013). Cross-cultural validation of the international affective picture system (IAPS) on a sample from Bosnia and Herzegovina. Psychologija, 46, 17-26.

Dufey, M., Fernández, A. M., \& Mayol, R. (2011). Adding support to cross-cultural emotional assessment: Validation of the international affective picture system in a Chilean sample. Universitas Psychologica, 10, 521-533.

Ekman, P., \& Matsumoto, D. (1993-2004). Japanese and Caucasian Facial Expressions of Emotion (JACFEE). Palo Alto, CA: Consulting Psychologists Press.

Fox, E., Griggs, L., \& Mouchlianitis, E. (2007). The detection of fearrelevant stimuli: Are guns noticed as quickly as snakes? Emotion, 7 , 691-696.

Gilet, A.-L., Grühn, D., Studer, J., \& Labouvie-Vief, G. (2012). Valence, arousal, and imagery ratings for 835 French attributes by young, middle-aged, and older adults: The French emotional evaluation list (FEEL). European Review of Applied Psychology, 62, 173-181.

Grühn, D., \& Scheibe, S. (2008). Age-related differences in valence and arousal ratings of pictures from the international affective picture system (IAPS): Do ratings become more extreme with age? Behavior Research Methods, 40, 512-521.

Hamann, S., Herman, R. A., Nolan, C. L., \& Wallen, K. (2004). Men and women differ in amygdala response to visual sexual stimuli. Nature Neuroscience, 7, 411-416.

Hinojosa, J. A., Carretie, L., Valcarcel, M. A., Mendez-Bertolo, C., \& Pozo, M. A. (2009). Electrophysiological differences in the processing of affective information in words and pictures. Cognitive, Affective, \& Behavioral Neuroscience, 9, 173-189.

Horan, W. P., Wynn, J. K., Kring, A. M., Simons, R. F., \& Green, M. F. (2010). Electrophysiological correlates of emotional responding in schizophrenia. Journal of Abnormal Psychology, 119, 18-30.

Karama, S., Lecours, A. R., Leroux, J. M., Bourgouin, P., Beaudoin, G., Joubert, S., \& Beauregard, M. (2002). Areas of brain activation in males and females during viewing of erotic film excerpts. Human Brain Mapping, 16, 1-16.

Kemp, A. H., Silberstein, R. B., Armstrong, S. M., \& Nathan, P. J. (2004). Gender differences in the cortical electrophysiological processing of visual emotional stimuli. NeuroImage, 21, 632-646.

Kensinger, E. A., \& Schacter, D. L. (2006). Processing emotional pictures and words: Effects of valence and arousal. Cognitive, Affective, \& Behavioral Neuroscience, 6, 110-126.

Lang, P. J., Bradley, M. M., \& Cuthbert, B. N. (1995-2008). International affective picture system (IAPS): Technical manual and affective ratings. Gainesville: University of Florida. Center for Research in Psychophysiology.

Lang, P. J., Bradley, M. M., \& Cuthbert, B. N. (1998a). Emotion, motivation, and anxiety: Brain mechanisms and psychophysiology. Biological Psychiatry, 44, 1248-1263. 
Lang, P. J., Bradley, M. M., Fitzsimmons, J. R., Cuthbert, B. N., Scott, J. D., Moulder, B., \& Nangia, V. (1998b). Emotional arousal and activation of the visual cortex: An fMRI analysis. Psychophysiology, 35, 199-210.

Lasaitis, C., Ribeiro, R. L., \& Bueno, O. F. A. (2008). Brazilian norms for the international affective picture system (IAPS) - Comparison of the affective ratings for new stimuli between Brazilian and NorthAmerican subjects. Jornal Brasileiro de Psiquiatria, 57(57), 270 275.

LeDoux, J. (1996). The emotional brain: The mysterious understanding of emotional life. New York: Simon \& Schuster.

Libkuman, T. M., Otani, H., Kern, R., Viger, S. G., \& Novak, N. (2007). Multidimensional normative ratings for the international affective picture system. Behavior Research Methods, 39, 326-334.

Lithari, C., Frantzidis, C. A., Papadelis, C., Vivas, A. B., Klados, M. A., Kourtidou-Papadeli, C., ... Bamidis, P. D. (2010). Are females more responsive to emotional stimuli? A neurophysiological study across arousal and valence dimensions. Brain Topography, 23, 27-40.

Liu, P., \& Pell, M. D. (2012). Recognizing vocal emotions in Mandarin Chinese: A validated database of Chinese vocal emotional stimuli. Behavior Research Methods, 44, 1042-1051.

Liu, T., Pinheiro, A. P., Deng, G., Nestor, P. G., McCarley, R. W., \& Niznikiewicz, M. A. (2012). Electrophysiological insights into processing nonverbal emotional vocalizations. Neuroreport, 23, 108112 .

Lohani, M., Gupta, R., \& Srinivasan, N. (2013). Cross-cultural evaluation of the international affective picture system on an Indian sample. Psychological Studies, 58, 233-241.

Lundqvist, D., Flykt, A., \& Öhman, A. (1998). The Karolinska Directed Emotional Faces -KDEF [CD ROM]. Department of Clinical Neuroscience, Psychology section, Karolinska Institutet.

Marchewka, A., Żurawski, Ł., Jednoróg, K., \& Grabowska, A. (2014). The Nencki affective picture system (NAPS): Introduction to a novel, standardized, wide-range, high-quality, realistic picture database. Behavior Research Methods, 46, 596-610.

McManis, M. H., Bradley, M. M., Berg, W. K., Cuthbert, B. N., \& Lang, P. J. (2001). Emotional reactions in children: Verbal, physiological, and behavioral responses to affective pictures. Psychophysiology, $38,222-231$.

Mesquita, B., \& Walker, R. (2003). Cultural differences in emotions: A context for interpreting emotional experiences. Behaviour Research and Therapy, 41, 777-793.

Mickley, K. R., \& Kensinger, E. A. (2008). Emotional valence influences the neural correlates associated with remembering and knowing. Cognitive, Affective, and Behavioral Neuroscience, 8, 143-152.

Mikels, J. A., Fredrickson, B. L., Larkin, G. R., Lindberg, C. M., Maglio, S. J., \& Reuter-Lorenz, P. A. (2005). Emotional category data on images from the international affective picture system. Behavior Research Methods, 37, 626-630.

Moltó, J., Montañés, S., Poy, R., Segarra, P., Pastor, M. C., Tormo, M. P., \& Vila, J. (1999). Un nuevo método para el estudio experimental de las emociones: El International Affective Picture System (IAPS). Adaptación española [A new method for the experimental study of emotions: The International Affective Picture System (IAPS). Spanish adaptation]. Revista de Psicología General y Aplicada, 52, 55-87.

Osgood, C. E., Suci, G., \& Tannenbaum, P. (1957). The measurement of meaning. Urbana, IL: University of Illinois Press.

Paulmann, S., \& Kotz, S. A. (2008). Early emotional prosody perception based on different speaker voices. Neuroreport, 19, 209-213.

Pessoa, L. (2009). How do emotion and motivation direct executive control? Trends in Cognitive Sciences, 13, 160-166.

Potter, M. C., Staub, A., \& O'Connor, D. H. (2004). Pictorial and conceptual representation of glimpsed pictures. Journal of Experimental Psychology: Human Perception and Performance, 30, 478-489.
Pinheiro, A. P., Del Re, E., Mezin, J., Nestor, P. G., Rauber, A., McCarley, R. W., \& Niznikiewicz, M. (2013a). Sensory-based and higher-order operations contribute to abnormal emotional prosody processing in schizophrenia: An electrophysiological investigation. Psychological Medicine, 43, 603-618.

Pinheiro, A. P., Liu, T., Nestor, P. G., McCarley, R. W., Goncalves, O. F., \& Niznikiewicz, M. A. (2013b). Visual emotional information processing in male schizophrenia patients: Combining ERP, clinical and behavioral evidence. Neuroscience Letters, 550, 75-80.

Ribeiro, R. L., Pompéia, S., \& Bueno, O. F. A. (2005). Comparison of Brazilian and American norms for the international affective picture system (IAPS). Revista Brasileira de Psiquiatria, 27, 208-215.

Russell, J. A. (1980). A circumplex model of affect. Journal of Personality and Social Psychology, 39, 1161-1178.

Sakaki, M., Niki, K., \& Mather, M. (2012). Beyond arousal and valence: The importance of the biological versus social relevance of emotional stimuli. Cognitive, Affective, \& Behavioral Neuroscience, 12, 115-139.

Schimmack, U. (2005). Attentional interference effects of emotional pictures: Threat, negativity, or arousal? Emotion, 5, 55-66.

Scott, G. G., O'Donnell, P. J., Leuthold, H., \& Sereno, S. C. (2009). Early emotion word processing: Evidence from event-related potentials. Biological Psychology, 80, 95-104.

Shao-Hua, H., Ning, W., \& Wen-Tao, G. (2005). Cross-cultural study of affective reactions of Chinese and American healthy adults. Chinese Journal of Clinical Psychology, 13, 265e266.

Silva, J. R. (2011). International affective picture system (IAPS) in Chile: A crosscultural adaptation and validation study. Terapia Psicológica, 29, 251-258.

Soares, A. P., Comesaña, M., Pinheiro, A. P., Simões, A., \& Frade, S. (2012). The adaptation of the affective norms for English words (ANEW) for European Portuguese. Behavior Research Methods, 44, 256-269.

Soares, A. P., Pinheiro, A. P., Costa, A., Frade, S., Comesaña, M., \& Pureza, R. (2013). Affective auditory stimuli: Adaptation of the international affective digitized sounds (IADS-2) for European Portuguese. Behavior Research Methods, 45, 1168-1181.

Tok, S., Koyuncu, M., Dural, S., \& Catikkas, F. (2010). Evaluation of international affective picture system (IAPS) ratings in an athlete population and its relations to personality. Personality and Individual Differences, 49, 461-466.

Van Berkum, J. J. A., De Goede, D., Alphen, P. M. V., Mulder, E. R., \& Kerstholt, J. H. (2013). How robust is the language architecture? The case of mood. Frontiers in Psychology, 4, 1-19.

Verschuere, B., Crombez, G., \& Koster, E. (2001). The international affective picture system: A Flemish validation study. Psychologica Belgica, 41, 205-217.

Vila, J., Sánchez, M., Ramírez, I., Fernández, M. C., Cobos, P., Rodríguez, S., ... Moltó, J. (2001). El Sistema Internacional de Imágenes Afectivas (IAPS): Adaptación española. Segunda parte [The International Affective Picture System (IAPS): Spanish adaptation. Second part]. Revista de Psicología General y Aplicada, 54, $635-657$.

Wrase, J., Klein, S., Gruesser, S. M., Hermann, D., Flor, H., Mann, K., ... Heinz, A. (2003). Gender differences in the processing of standardized emotional visual stimuli in humans: a functional magnetic resonance imaging study. Neuroscience Letters, 348, 41-45.

Yi, J.-Y., Liu, M.-F., Luo, Y.-Z., \& Yao, S.-Q. (2006). Gender differences in the reaction to emotional pictures. Chinese Journal of Clinical Psychology, 14, 583-585.

Yu, J., Hung, D. L., Tseng, P., Tzeng, O. J. L., Muggleton, N. G., \& Juan, C. H. (2012). Sex differences in how erotic and painful stimuli impair inhibitory control. Cognition, 124, 251-255.

Yuxia, H., \& Yuejia, L. (2004). Native assessment of international affective picture system. Chinese Mental Health Journal, 18, 631-634. 